REVISTA

MEXICANA DE

ECONOMÍA Y

FINANZAS

REMEF

(THE MEXICAN JOURNAL OF

ECONOMICS AND FINANCE)
Revista Mexicana de Economía y Finanzas, Nueva Época

Volumen 16 Número 1, Enero - Marzo 2021, pp. 1-35, e479

DOI: https://doi.org/10.21919/remef.v16i1.479

(Recibido: 17/marzo/2020, aceptado: 2/julio/2020, publicado: $12 /$ noviembre/2020)

\title{
Viabilidad de introducir contratos de derivados de gas natural en el Mercado Mexicano de Derivados: Un enfoque Hubbert-Grey
}

\author{
Luis Enrique García-Pérez ${ }^{1}$ - Instituto Politécnico Nacional, México \\ Francisco Ortiz Arango ${ }^{2}$ - Universidad Panamericana, México \\ Salvador Cruz Aké ${ }^{3}$ - Instituto Politécnico Nacional, México
}

A partir de la promulgación de la reforma energética de 2013 en México, se abrió la posibilidad de incorporar en el Mercado Mexicano de Derivados (MexDer) contratos de derivados de gas natural. El objetivo de esta investigación es examinar la viabilidad de introducir contratos de derivados de gas natural en el MexDer. Para ello, la proyección de la oferta de gas natural se calcula con el modelo de Hubert, y la de la demanda se obtiene mediante una novedosa combinación de los modelos de Grey y de Vasicek. Posteriormente mediante simulación Monte Carlo se calculan las primas de un contrato de futuros y uno de opciones europeas sobre el precio del gas natural, los cuales fueron diseñados acorde a la normatividad del MexDer. Los resultados obtenidos muestran que por el momento dichos contratos no son viables, fundamentalmente por el reducido volumen de producción para los posibles contratos, en comparación con la creciente demanda, por lo que se recomienda impulsar la inversión en la plataforma productiva y comercial de modo que la transacción de derivados de gas natural sea viable

Clasificación JEL: C22, C63, L19, L71, Q31, Q35, Q43.

Palabras clave: Reforma energética, gas natural, contratos de derivados, MEXDER.

\section{Feasibility of Introducing Natural Gas Derivatives Contracts in the Mexican Derivatives Market: A Hubbert-Gray Approach}

After the enactment of the 2013 energy reform in Mexico, the possibility of incorporating natural gas derivative contracts into the Mexican Derivatives Market (MexDer) was opened. The aim of this research is to examine the feasibility of introducing natural gas derivative contracts in the MexDer. For this, we use the Hubert model to calculate the projection of the natural gas supply, and the demand projection is obtained through a novel combination of the Gray and the Vasicek models. Subsequently, employing Monte Carlo simulation, we calculate the premiums of a futures contract and a contract of the European options over the price of natural gas, both were designed according to the regulations of the MexDer. The results obtained show that at the moment such contracts are not viable, mainly due to the reduced volume of production for possible contracts, compared to the growing demand, so it is recommended to promote investment in the productive and commercial platform so that the natural gas derivatives transaction is viable.

JEL Classification: C22, C63, L19, L71, Q31, Q35, Q43.

Keywords: Energy reform, natural gas, derivative contracts, MEXDER

1 ORCID: https://orcid.org/0000-0002-1836-9709

2 Autor de correspondencia. ORCID: https://orcid.org/0000-0003-2707-4459

3 ORCID: https://orcid.org/0000-0003-1452-377X

*Sin fuente de financiamiento para el desarrollo de la investigación. 


\section{Introducción}

El consumo de gas natural se ha incrementado de manera significativa en los países pertenecientes a la OCDE en las últimas décadas (Frauendorfer, 2007). La liberación paulatina en el mercado internacional, así como el desarrollo tecnológico en su transportación han abierto barreras en la comercialización de gas natural alrededor del mundo (Amell et al., 2016).

En México, la reforma energética de 2013, incluyó una modificación constitucional que permite la participación del sector privado en el proceso desde la exploración hasta la enajenación del hidrocarburo (SENER, 2014). La liberación del sector energético ha traído cambios en la dinámica de tal mercado y por tanto nuevos riesgos, por lo que es conveniente incluir en este mercado el uso de varias herramientas de administración de riesgos.

Soldo (2012) sugiere qué, con el desarrollo de los sistemas de distribución de gas natural y consumo del mismo, los agentes involucrados se han planteado preguntas como: ¿Cuánto gas se consume? ¿Por cuánto tiempo habrá gas natural?, entre otras. El cálculo de estos pronósticos es una de las principales tareas a desarrollar en este sector, en este trabajo se plantean alternativas para este propósito.

En la presente investigación se calcula el pronóstico de la demanda de gas natural mediante el modelo de Grey GM(1,1) (Xie y Li, 2009), (Hsu and Chen, 2003), a través de la dinámica seguida por el consumo de gas natural. Utilizando el modelo de Hubbert se obtiene el pronóstico de la oferta a partir del comportamiento histórico de la extracción del gas natural. Adicionalmente, se utiliza el modelo de Ornstein-Uhlenbeck para calcular la tasa de consumo de gas natural, para lo cual se supone que existe una dinámica de reversión a la media hacia un valor constante. Finalmente se simulan un contrato de futuros y uno de opciones europeas con los cuales se plantea una estrategia de cobertura de riesgo para la adquisición de gas natural.

El presente artículo se desarrolla de la siguiente manera: En la sección dos, se analizan los antecedentes y el contexto de las condiciones de oferta y demanda del mercado de gas natural, tanto en México como en el exterior. Se plantea brevemente la comercialización de derivados de gas natural en mercados foráneos. En la tercera sección, se describen los modelos utilizados para pronosticar la demanda y la oferta de gas natural, ya mencionados anteriormente, así como los elementos fundamentales de la operación de un mercado de derivados. En la sección cuatro, se muestran los resultados obtenidos con los modelos expuestos de la sección tres, así mismo, basados en los precios de las primas tanto del contrato de futuros como el de una opción europea sobre el precio de gas natural, calculados mediante simulación Monte Carlo; se diseñan contratos de futuros y de una opción europea para el precio de gas natural, considerando las disposiciones técnicas señaladas por el Mercado Mexicano de Derivados (MexDer). Finalmente se plantean las conclusiones sobre los resultados obtenidos.

\section{Antecedentes y contexto del mercado del gas natural}

El gas natural es una de las principales fuentes de energía primaria, utilizado en diversos sectores de la economía. En este trabajo se considerarán a los sectores eléctrico, industrial y petrolero, debido a 
que la demanda de gas natural en estos sectores representa el $98.3 \%$ del consumo total en México, según SENER (2017).

De manera muy sintetizada, el ciclo completo, desde la producción hasta el consumo del gas natural se puede representar mediante el siguiente esquema:

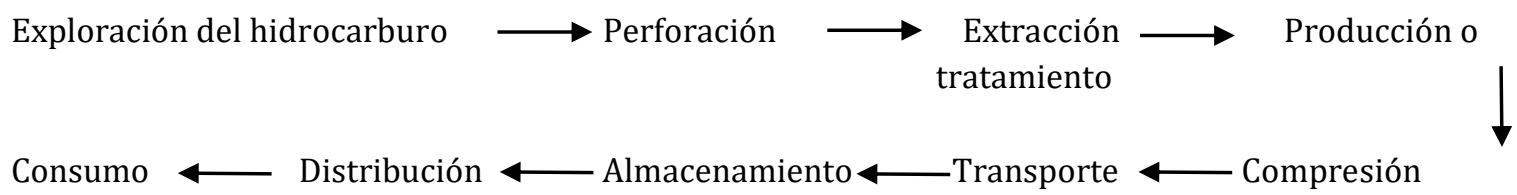

En el cual podemos identificar la participación de los siguientes agentes en el mercado:

1. Productores de gas natural (exploradores y extractores)

2. Compañías de ductos (transportadores)

3. Compañías de entrega locales (distribuidores)

4. Consumidores

\subsection{Comercio de gas natural a nivel mundial}

Amell et al. (2016) señalan que la manera más usual de transportar gas natural en trayectos largos es en forma gas natural licuado (GNL) a través de ductos, el cual se encuentra a una temperatura aproximada de $-161^{\circ} \mathrm{C}^{4}$, y cuando llega a su destino final se re-gasifica y se envía a través de otro sistema de ductos para su distribución y consumo final. Frauendorfer (2007) sugiere que el mercado mundial se divide en 3 grandes regiones:

- Norte América, donde las mayores transacciones se realizan entre Canadá y Estados Unidos.

- Europa, donde las principales transacciones van desde Argelia, Noruega y Rusia hacia Europa Occidental.

- Asia, donde las ventas más importantes son conectadas en Asia del Este, Australia e Indonesia.

Se considera que los mercados más influyentes se localizan en el Reino Unido y en los Estados Unidos, cuyos precios se establecen mediante mecanismos competitivos que responden a las fuerzas del mercado. A diferencia de países como Rusia, donde la empresa del estado (Rosneft) establece los precios del hidrocarburo de manera monopólica.

Frauendorfer (op. cit.) menciona que las naciones pertenecientes a la OCDE, han tenido una evolución similar respecto al consumo de gas natural, por ejemplo, en Estados Unidos considerando el periodo de 1949 a 2019, hubo una tendencia creciente en el consumo anual de gas natural hasta principios de los años setenta, después, desde ese periodo hasta mediados de la década de 1980 el consumo anual disminuyó, sin embargo, se registró una tendencia alcista desde entonces a la fecha.

En cuanto a la perspectiva para el consumo futuro de gas natural, el Chicago Mercantil Exchange (CME) menciona que el gas natural es actualmente la tercera fuente de energía más

\footnotetext{
${ }^{4}$ Esto permite comprimir al gas natural alrededor de 600 veces el volumen que tiene a temperatura ambiente y presión atmosférica, por lo cual se optimiza el uso de los ductos en su transportación.
} 
importante, después del petróleo y el carbón, por lo que se espera que crezca rápidamente al ser considerado el combustible de la "transición energética", y que supere al carbón en segundo lugar para 2030 debido a su uso en la generación de electricidad.

\subsection{El mercado de derivados en el comercio de gas natural}

El principal objetivo de la comercialización de contratos de derivados es la cobertura de riesgos, estos son utilizados por los agentes económicos que desean mitigar el riesgo de cambios adversos en los precios de los activos que poseen en el mercado de contado o físico. Dos de los principales contratos de derivados comercializados en los mercados regulados son los de futuros y los de opciones. 5

Estados Unidos fue el primer país en negociar contratos de futuros sobre gas natural, después de que en 1989 se desreguló el precio del gas natural (Aguirre et al., 2012). En la actualidad, la estructura del mercado de gas natural es eficiente, transparente y líquida con una gran cantidad de participantes, donde existen varios centros de comercialización como: New York City Gate, Chicago City Gate y Katy $H u b$, pero sin duda el predominante es Henry Hub, el cual que se ha convertido en el nodo que fija el precio de referencia para el gas natural a nivel mundial.

El desarrollo de grandes centros de negociación de gas natural implica que se acondicione infraestructura donde existan intersecciones de ductos principales y acceso adecuado a las instalaciones de almacenamiento. En Europa occidental, se encuentran varios nodos donde se fijan los precios del gas natural, destacando el National Balancing Point (NBP) en el Reino Unido y el Title Transfer Facility (TTF) en Holanda.

Esto es importante, dado que, en su mayoría, estos centros de comercialización son los puntos de entrega de las transacciones de contratos de futuros en bolsas como New York Mercantile Exchange (NYMEX), Intercontinental Exchange (ICE), European Energy Exchange (EEX) y Natural Gas Exchange (NGX). Por el volumen de contratos negociados, NYMEX es el mayor mercado de futuros de gas natural a nivel mundial.

Henry Hub cumple una serie de ventajas entre las que destacan la ubicación estratégica y la infraestructura logística, por lo que se ha vuelto el "fijador" del precio de referencia, cuando los mercados locales ponen precio a su gas natural, tienden a hacerlo con base en un diferencial con Henry $H u b$. Este diferencial se determina por las condiciones del mercado regional, los costos de transporte y la capacidad de transmisión disponible entre ubicaciones. A diferencia del mercado físico, el mercado de futuros ofrece un descubrimiento de precios a lo largo del tiempo reflejado en la estructura de plazos, lo que permite estimar las expectativas del mercado para los precios futuros del gas natural.

En México, el MexDer es una filial del Grupo Bolsa Mexicana de Valores (BMV), inició operaciones el 15 diciembre de 1998, está constituido como una sociedad anónima de capital variable, autorizada por la Secretaría de Hacienda y Crédito Público (SHCP). Tanto el MexDer como su Cámara de Compensación, Asigna, son entidades autorreguladas que funcionan bajo la supervisión de la SHCP, el Banco de México y la Comisión Nacional Bancaria y de Valores. Actualmente el MexDer opera 37 contratos de futuros: 2 de divisas, 2 de índices, 18 de instrumentos de deuda y 15 de

\footnotetext{
${ }^{5}$ Véase también Ibañez et al. (2016).
} 
acciones; en el caso de las opciones, existen 17 contratos: 1 de divisas, 1 de índices y 15 de acciones, (MexDer, 09/11/2019). Como se puede ver ninguno dedicado a productos energéticos.

\subsection{Contexto del mercado del gas natural en México}

El crecimiento de la industria del gas natural en México se atribuye principalmente al desarrollo industrial que experimentó el país a partir de la década de 1940. A pesar del crecimiento sostenido, a finales de los setentas, la producción de Petróleos Mexicanos (Pemex) comenzó a decaer y con esto los ingresos netos, así como las reservas, situación que provocó una crisis de la propia empresa (Palacios, 1987), tendencia que cambió súbitamente a inicios de la década de 1980. En este contexto, Pemex ha operado bajo dos políticas de precios: la primera, que va desde sus inicios hasta 1982, caracterizada por el establecimiento de precios bajos y subsidios; y la segunda, iniciada en 1982, en la que destaca el intento de eliminar los subsidios y fijar los precios de acuerdo a la situación económica y financiera del país.

Debe hacerse notar, que debido al inicio de la explotación del yacimiento de Cantarell ${ }^{6}$ en 1979, se presentó a partir de ese momento un acelerado crecimiento en la producción petrolera, que alcanzó su máximo de producción en 2008 con alrededor de 3.43 millones de barriles diarios, sin embargo, a partir de 2009 inició el descenso de la producción, hasta llegar en la actualidad a un promedio de 1.7 millones de barriles diarios. Algo similar ocurrió con la producción de gas natural, la cual alcanzó su producción máxima en 2009 con alrededor de 7,030 millones de pies cúbicos diarios (SENER, 2017) y a partir de allí descendió hasta el valor actual de alrededor de 4,850 millones de pies cúbicos diarios (SIE).

El año 1995, se puede considerar como el inicio de la liberación del mercado de gas natural, al implementarse una primera reforma al mercado del gas natural. Se realizaron modificaciones al artículo 27 constitucional, referentes a la actividad económica del gas natural, se creó la Comisión Reguladora de Energía (CRE), institución encargada de regular la operación del mercado de gas natural (SENER, 2016). A partir de este año, el sector privado podía llevar a cabo actividades de transportación, almacenamiento y distribución de gas natural, así como su participación en la construcción, propiedad y operación de la infraestructura asociada a dichas actividades, celebrando contratos con Petróleos Mexicanos.

En 2008, se implementó una nueva regulación con objeto de otorgar a la Secretaría de Energía (SENER) y la Comisión Reguladora de Energía (CRE) responsabilidades sobre política energética (Miller et al., 2015). Se definió la venta de primera mano (VPM) y la CRE fue el organismo encargado de establecer las condiciones de VPM, además de determinar las zonas geográficas de distribución entre otros asuntos (SENER, 2017).

Pese a la relevancia del sector energético en la economía nacional, los problemas que sufría Pemex desde décadas atrás dieron origen a la propuesta de la reforma energética: la caída persistente en las reservas totales, una menor producción de hidrocarburos, así como pérdidas financieras netas. El Gobierno Federal identificó la inconveniencia de que Pemex continuara asumiendo la totalidad de los riesgos inherentes a las actividades de exploración y extracción, la insuficiente producción de refinados, gas, petroquímicos e infraestructura de transporte. También se argumentó que en el país

\footnotetext{
${ }^{6} \mathrm{El}$ yacimiento de Cantarell es considerado como el segundo yacimiento más grande en la historia.
} 
los recursos prospectivos se encontraban, en su mayoría, en yacimientos de gran complejidad (no convencionales y aguas profundas) que demandaban capacidades de ejecución e inversión que sólo se podrían alcanzar con la participación de empresas del sector privado (Miller et al., 2015).

\subsection{La reforma energética de 2013}

Se reconoce la reforma energética de 2013 como parte de un esfuerzo para modernizar y diversificar la economía del país, aumentar la competitividad de la industria. Su objetivo es incentivar la productividad del sector energético mediante la inversión privada. Propiciar un sector sostenible, eficiente, transparente y productivo, aumentar los beneficios derivados de los hidrocarburos del país. (IEA, 2016)

En cuanto a la regulación del mercado de gas natural, la reforma energética fue un conjunto de cambios a los artículos constitucionales 25,27 y 28, donde se permite la participación del sector privado en cada parte del proceso productivo, aunque en el corto plazo Pemex seguirá siendo el principal productor del país.

La reforma energética de 2013 fue una reforma constitucional, en la cual se realizaron modificaciones en el marco legal, otorgando nuevas atribuciones a las instituciones reguladoras del sector energético y se crean otras con el fin de tener actividades de regulación complementarias, algunos de los planteamientos más significativos son:

- SENER mantiene sus facultades para definir la política energética, conceder asignaciones a Pemex, elegir cuales áreas son objeto de contratos de exploración y extracción, con asistencia técnica de la Comisión Nacional de Hidrocarburos ( $\mathrm{CNH}$ ), además de aprobar y publicar el plan quinquenal de licitaciones, criterios y mecanismos de adjudicación.

- La CNH y la CRE son los órganos reguladores en materia de energía, con personalidad jurídica propia, autonomía técnica y de gestión. Además, la CRE regulará y otorgará permisos para el almacenamiento, transporte y distribución por ductos; regular el acceso a terceros a los ductos de almacenamiento y transporte, establecer las disposiciones a que deben sujetarse los permisionarios y usuarios del hidrocarburo con el fin de promover el desarrollo del mercado y su competitividad.

- El Centro Nacional de Control de Gas Natural (CENAGAS) es el gestor y administrador independiente del Sistema de Transporte y Almacenamiento Nacional Integrado de Gas Natural (SISTRANGAS), al mismo tiempo se encarga de operar y mantener la infraestructura de transporte, de cuyos permisos es titular. Adicionalmente, el CENAGAS es quien propone a la SENER, para su aprobación, previa opinión técnica de la CRE, el Plan Quinquenal de Expansión del SISTRANGAS.

- La SENER está facultada para establecer las condiciones económicas de licitaciones y contratos relativos a los términos fiscales que permitan a la nación obtener ingresos que contribuyan a su desarrollo.

- Pemex se conforma como una empresa productiva del Estado (EPE) de propiedad exclusiva del Gobierno Federal, con personalidad jurídica, patrimonio propio y con autonomía técnica, operativa y de gestión; sujeta a su ley y disposiciones que deriven de los mismos. 
En cuanto a la liberalización de los precios del gas natural, se propuso que la metodología de Venta de Primera Mano (VPM) continuara hasta el 16 de junio de 2017, por lo que se publicó en el Diario Oficial de la Federación (DOF) el acuerdo número A/026/2017, informando sobre la eliminación del método VPM en el país con excepción de la zona Sur, en tanto se logre mayor competitividad en la región. Se consideró que la CRE eliminara la fórmula del precio de VPM en todo el país para 2018 y que el precio se determine bajo condiciones de libre mercado. A partir del 20 de marzo de 2018, la CRE incluyó la zona sur.

De acuerdo a esto, la CRE elaborará un Índice de Referencia Nacional de Precios de Gas Natural al mayoreo (INPGN), el cual resulte del precio de las transacciones pactadas. El INPGN es publicado en Pesos por Giga joule (MXN/GJ) y en Dólares por Millón de unidades térmicas británicas (USD/MBtu), esto con el fin de hacerlo comparable con los precios e índices de referencia internacionales. La publicación de dicho índice de precios será transitoria, hasta que se alcancen condiciones de competencia en el mercado de gas natural.

\subsection{Demanda, oferta e importación de gas natural en México} Demanda.

El gas natural se ha convertido en la principal fuente de consumo de energía, tanto en el sector eléctrico, así como el industrial y el petrolero, su conveniencia radica en su bajo costo en comparación con otros energéticos, como el combustóleo, coque de petróleo o carbón (SENER, 2017), y adicionalmente una menor generación de contaminantes debido a su uso, esto ha promovido un incremento sustancial en su demanda.

Oferta.

Según SENER (2016), en 2015, las regiones norte y sur de México, fueron las principales abastecedoras de gas natural, con una producción conjunta que representó $65.7 \%$ de la producción nacional. Cabe mencionar que la producción de gas natural no asociado en México ha decrecido, debido a la menor rentabilidad obtenida como consecuencia de los bajos precios del gas natural en Estados Unidos (SENER, 2015).

Respecto a la comercialización de gas natural, de acuerdo a SENER (2016), en 2018 Pemex terminaría el Programa de Cesión de Contratos (PCC), tal programa establece que Pemex deberá ceder contratos de suministro de gas natural, hasta tener el 30\% de participación en la comercialización, bajo la aprobación de la CRE. Para marzo de 2018, Pemex había terminado la fase I, cediendo un $30.03 \%$ de sus contratos, mientras que, en septiembre de 2018, la CRE aprobó que las fases II y III se realicen en una sola fase final con el propósito de otorgar aproximadamente un 25.12\% de los contratos. Con la eliminación de la fórmula de VPM y el PCC, se busca incentivar la producción. Importación.

Debido a que la demanda ha venido superando de manera constante a la oferta nacional, México se ha visto en la necesidad de incrementar la importación de gas natural para lograr cubrir el déficit que se tiene. 


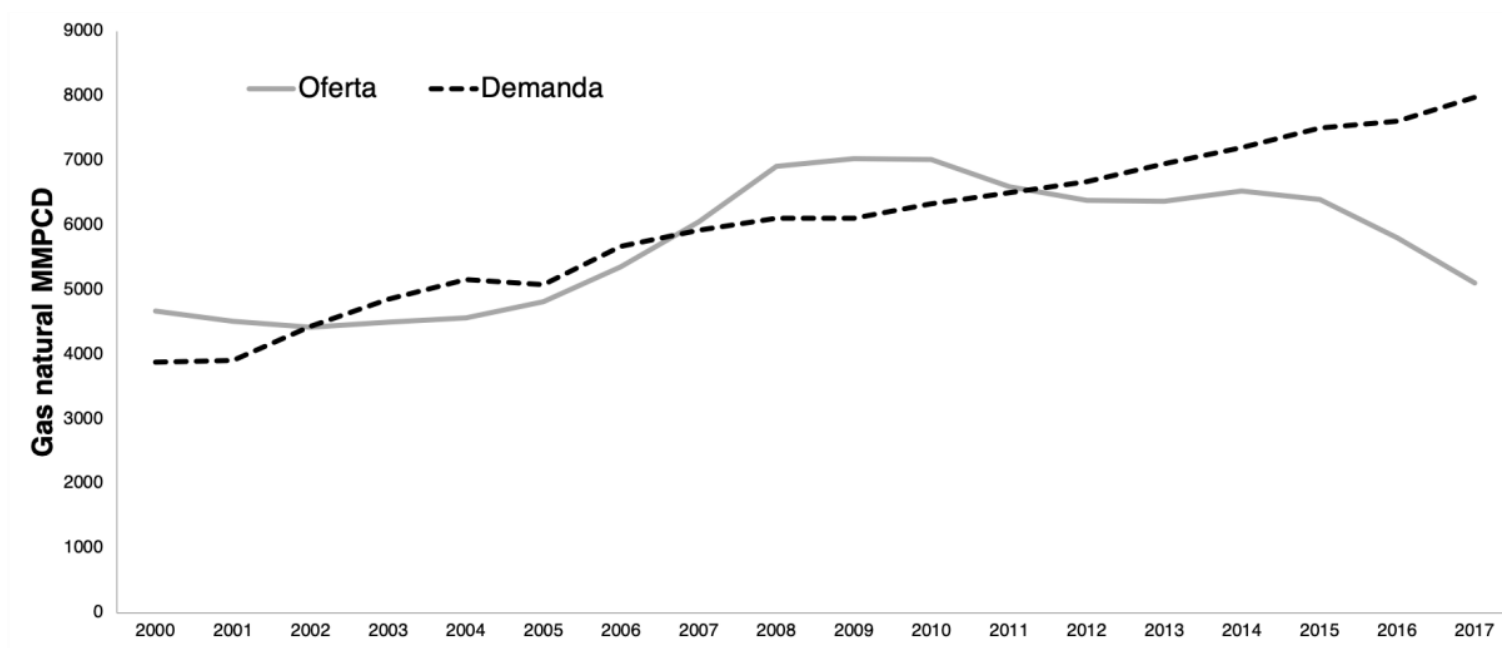

Figura 1. Oferta y demanda de gas natural (MMPCD)

Fuente: Sistema de Información Energética

Como se aprecia en la figura 1, las importaciones de gas natural son resultado de la creciente brecha entre oferta nacional y demanda, donde el desabastecimiento es compensado por medio de importaciones, principalmente mediante gasoductos conectados a los Estados Unidos en la frontera norte del país.

\section{Descripción y fundamentos teóricos de los modelos utilizados}

\subsection{Modelo de Grey de primer orden de una variable GM(1,1)}

El modelo GM(1,1) es un método de elaboración de pronósticos no estadístico que es particularmente efectivo cuando el número de observaciones es insuficiente, el cual se puede utilizar para predecir el comportamiento de series de tiempo no lineales. Para su desarrollo se define inicialmente a la serie de tiempo observada como:

$x^{(0)}=\left(x^{(0)}(1), x^{(0)}(2), \ldots, x^{(0)}(n)\right)$, donde $n$ es el número de observaciones. Y posteriormente se define a la serie de tiempo $x^{(1)}=\left(x^{(1)}(1), x^{(1)}(2), \ldots, x^{(1)}(n)\right)$, donde:

$$
\begin{gathered}
x^{(1)}(1)=x^{(0)}(1) \\
x^{(1)}(k)=\sum_{m=1}^{k} x^{(0)}(m), \quad k=2,3, \ldots, n .
\end{gathered}
$$

El modelo GM(1,1) se construye estableciendo una ecuación diferencial de primer orden para $x^{(1)}(t):$

$$
\frac{d x^{(1)}(t)}{d t}+a x^{(1)}(t)=b
$$


La obtención de los parámetros $a$ y $b$ de la ecuación (1) se realiza mediante el método de mínimos cuadrados ordinarios (MCO).

$$
\hat{x}^{(1)}(t)=\left(x^{(0)}(1)-\frac{\hat{b}}{\hat{a}}\right) e^{-\hat{a} t}+\frac{\hat{b}}{\hat{a}} .
$$

A través de la ecuación (2), conocida como IAGO (inversa de la solución de la ecuación diferencial) se obtienen las estimaciones de GM $(1,1)$, donde $\hat{x}^{(1)}(\mathrm{t})$ es el valor pronosticado de GM $(1,1), \mathrm{x}^{(0)}(1)$ es el primer dato de la serie, $\hat{a}$ y $\hat{b}$ son los parámetros estimados. Mientras que $x^{(1)}(t+1)$ es el pronóstico de consumo y se calcula mediante:

$$
x^{(1)}(t+1)=\hat{x}^{(1)}(t+1)-x^{(1)}(t) .
$$

La ecuación diferencial en la que se basa el modelo GM(1,1) es determinista e implica una dinámica siempre creciente de la demanda de gas. Este supuesto no es necesariamente correcto en el corto plazo, pues factores ambientales o económicos pueden alterar aleatoriamente los consumos individuales.

Para subsanar estas deficiencias, se propone el uso de un modelo estocástico con reversión a la media que capte las desviaciones aleatorias respecto a las tendencias (crecientes o no) de largo plazo, el cual se plantea a continuación.

\subsection{Modelo de reversión a la media}

Es un modelo que se aplica en el caso donde la dinámica de una tasa corta presenta reversión a la media hacia un valor constante. En este caso $r_{t}$ es la tasa de crecimiento de la demanda de gas natural y se supone que $r_{t}$ es conducida por una ecuación diferencial estocástica (Vasicek, 1977) de la forma.

$$
d r_{t}=a\left(b-r_{t}\right) d t+\sigma d W_{t}
$$

La ecuación diferencial que guía el cambio en la tasa de crecimiento de la demanda de gas permite modelar pequeñas desviaciones (brownianas) respecto a la demanda de largo plazo (el modelo debe recalibrarse con el paso del tiempo). Es importante hacer notar que la solución de la ecuación diferencial genera una estructura de plazo para la demanda de gas que cumple con las características deseables de la obtenida a partir de los precios futuros.

Esta característica es uno de los resultados más interesantes del trabajo, pues permite vincular los precios futuros del hidrocarburo con un modelo reducido recalibrable.

En este trabajo, se hará uso de modelos de simulación Monte Carlo para hacer estimaciones sobre la demanda de gas natural basadas en este modelo de reversión a la media, para posteriormente, compararlas a las demandas reales.

La solución de la ecuación (4), es la siguiente:

$$
r_{t}=b+\left(r_{0}-b\right) e^{-a t}+\sigma \int_{0}^{t} e^{-a(t-s)} d W(s)
$$


Donde el valor obtenido al resolver la integral estocástica, es de la forma:

$$
\hat{r}(t)=b+\left(r_{0}-b\right) e^{-a t}+\frac{\sigma e^{-a t} \sqrt{e^{2 a t}-1}}{\sqrt{2 a}} Z .
$$

Una vez que se han estimado los parámetros $a, b, \sigma$ y generando diferentes valores de $\mathrm{Z}$ (diferentes valores de probabilidad para una variable estándar que se distribuye con media 0 y varianza 1), se obtienen estimaciones de $r_{t}$ para la demanda de gas natural. ${ }^{7}$

\subsection{Modelo de curva de Hubbert}

Como sabemos el gas natural es un recurso finito, por lo cual es fundamental poder calcular el pico de máxima producción, la producción acumulada y el tiempo en el que esto ocurrirá. En 1956 M. King Hubbert (1956) desarrolló un modelo matemático mediante el cual es posible calcular estos valores, la ecuación diferencial del modelo de Hubbert es de la forma:

$$
P_{t}=\frac{d Q_{t}}{d t}=a Q_{t}-b Q_{t}^{2}
$$

Aunado a esta ecuación, Hubbert diseñó un método que permite generar por extrapolación las gráficas de producción anual histórica del recurso en cuestión (petróleo, gas o uranio, por ejemplo), este método se basa en dos condiciones:

1. Las condiciones de frontera:

a. En el tiempo inicial $t=0$, la producción acumulada $Q_{t}=0$ y la tasa de producción $P_{t}$ también es cero, es decir $P_{t}=\frac{d Q_{t}}{d t}=0$.

b. En el tiempo final $t=t_{f}$, se tiene la producción acumulada total $Q$ y la tasa de producción $P_{t}$ vuelve a ser cero, es decir $P_{t}=\frac{d Q_{t}}{d t}=0$.

2. Por el teorema fundamental del cálculo se garantiza que si una función $y=f(x)$ es continua en un intervalo dado, entonces el valor de la integral definida de dicha función en tal intervalo será único y equivale al valor del área bajo la curva descrita por la función en el intervalo dado.

Estas condiciones fueron descritas por M. King Hubbert (1956) y se han aplicado a varios recursos finitos. Al punto de máxima producción se le conoce como "Pico de Hubbert", el cual tiende a coincidir con el punto medio de agotamiento del recurso no renovable. Esto significa que cuando se llega al Pico de Hubbert, se ha utilizado la mitad de todo el recurso extraído que existía en ese espacio. Conviene mencionar que este modelo se utiliza para predecir la producción futura, mas no el precio del gas natural.

A partir de la información sobre la cantidad producida de gas natural, Hubbert (1956) mostró una forma de construir un gráfico que convierte una curva logística en una línea recta, donde la tasa

\footnotetext{
${ }^{7}$ Véase, por ejemplo, Venegas-Martínez (2008)
} 
de producción está en función de la cantidad extraída previamente. Esta técnica se conoce como linealización de Hubbert.

Para aplicar esta técnica el primer paso es realizar la linealización de Hubbert, que es la base para obtener la curva de Hubbert. Se inicia tomando los datos de la producción acumulada $Q_{t}$, la cual se calcula mediante la suma de los datos históricos de producción de gas natural en el año $t$. Posteriormente tomamos los valores de la tasa de producción anual $P_{t}$, los cuales se dividen entre la producción acumulada para formar el cociente $P_{t} / Q_{t}$, a partir de (7) obtenemos:

$$
\frac{P_{t}}{Q_{t}}=a-b Q_{t}
$$

con la cual es posible llevar a cabo la regresión para la linealización de Hubbert. Los parámetros a y $b$ se obtienen mediante el método de mínimos cuadrados ordinarios, los cuales se sustituyen en la expresión $(9)^{8}$ para encontrar el valor de la producción final acumulada (Bere, 2010):

$$
\frac{a}{b}=Q
$$

Este valor nos proporciona la estimación de la cantidad total de gas natural disponible durante la vida del yacimiento. Por otro lado, sustituyendo (9) en (8) y despejando $P_{t}$ (Bere, 2010), se obtiene la expresión para calcular la producción anual pronosticada:

$$
P_{t}=Q_{t} a\left(\frac{1-Q_{t}}{Q}\right)
$$

donde:

$P_{t}$ es la tasa de producción anual pronosticada

$Q_{t}$ es la producción acumulada

$a$ es el ritmo de extracción

$Q$ es la cantidad total de gas natural disponible o producción acumulada total.

\subsection{Teoría del mercado de derivados}

\subsubsection{Futuro con activo subyacente gas natural}

El precio que se determina en el contrato de futuro, se le denomina precio teórico, el cual expresa el valor del activo mediante una estimación matemática. De acuerdo al tipo de activo subyacente se puede ocupar una fórmula particular para hallar el precio teórico, en el caso del precio teórico de un futuro con activo subyacente gas natural, la referencia además del precio spot, es incluir el costo de almacenamiento, la tasa de interés de libre riesgo y una tasa de conveniencia. El método de valuación se realiza en términos de una tasa continuamente capitalizable.

$$
F_{t}=S_{t} e^{\left(r_{f}+u-y\right)(T-t)}
$$

\footnotetext{
${ }^{8}$ La expresión (9) se obtiene al aplicar las condiciones de frontera a la expresión (7) y despejar de ésta a $Q$.
} 
$F_{t:}$ Precio teórico del contrato.

$S_{t}$ : Precio spot del subyacente.

$r_{f:}$ Tasa de interés libre de riesgo.

$u$ : Costo de almacenamiento por año como porcentaje del precio del subyacente.

$y$ : Tasa de conveniencia.

$T-t$ : Periodo de tiempo hasta el vencimiento del contrato.

Nótese que el precio teórico del contrato de futuros de gas natural tiene relación directa con la tasa de interés y el costo de almacenamiento, es decir, aumentos en el costo de almacenamiento y tasa de interés, incrementan el precio teórico, mientras que existe una relación inversa con la tasa de conveniencia. La tasa de conveniencia, se explica como el beneficio o prima, asociado con la posesión del producto subyacente, en lugar del contrato. El valor de la tasa de conveniencia no se puede calcular directamente y sólo tiene un valor implícito, lo que significa que depende de la probabilidad de eventos futuros. El agente que supone que el abastecimiento de gas natural será insuficiente, comprará el activo físico, aprovechando la tasa de conveniencia que genere el incremento del precio del gas natural como consecuencia del desabasto. Por ejemplo, si: $S_{t} e^{(y)(T-t)}>$ $S_{t} e^{\left(r_{f}+u\right)(T-t)}$ conviene mantener el activo físico, caso contrario se prefiere la adquisición del contrato de futuros.

\section{Marginación del contrato de futuros.}

Una vez definido el precio teórico del contrato de futuros, la cámara de compensación al ser la contraparte en las transacciones, debe garantizar la reducción del riesgo asociado a las transacciones de futuros y opciones. Para lograr tal objetivo, utiliza la Aportación Inicial Mínima (AIM), la cual cubre la pérdida máxima potencial de acuerdo al horizonte de liquidación para un portafolio de futuros. El mecanismo por el que logra establecer un precio justo a tal AIM es mediante el Theoretical Intermarket Margin System (TIMS) que es un modelo de marginación estándar aplicado en los mercados de derivados internacionales.

La AIM para futuros se basa en tres componentes para la protección ante eventos adversos en el mercado (AIM riesgo, AIM opuesta y AIM entrega); sin embargo, suele haber una sustitución entre la AIM por entrega y la AIM por riesgo, dependiendo del procedimiento de liquidación (físico o financiero). En el caso del contrato de futuros de gas natural, los componentes son la AIM opuesta y la AIM por entrega.

La AIM opuesta se calcula en función a la variación máxima esperada (vme), al tamaño del contrato y al coeficiente de determinación entre las distintas series (Díaz-Tinoco y Venegas-Martínez, 2004), usando la expresión:

$$
\alpha=(v m e) T(1-\omega) .
$$

La AIM por entrega, se determina considerando la vme, el tamaño del contrato y el factor de liquidez del período de liquidación que se calcula a través de la agregación del tiempo a la volatilidad diaria, raíz del número de días entre la fecha de liquidación y la fecha de vencimiento, es decir: 


$$
\alpha_{E}=(v m e) T \sqrt{n} \text {. }
$$

En la ecuación (13) la vme define la máxima fluctuación posible al alza o a la baja en el precio del activo subyacente para un horizonte de tiempo, donde $z$ es el nivel de confianza, $\sigma$ es la volatilidad diaria de los rendimientos del activo subyacente, $T$ es la agregación en el tiempo a la volatilidad diaria y $P$ es el precio del activo subyacente, vme se calcula así:

$$
v m e=z \sigma P \sqrt{T}
$$

Opción con activo subyacente gas natural.

En la práctica las opciones sobre gas natural operan como cualquier otro tipo de opción, existen una gran variedad de modelos para determinar el precio de las opciones o prima, que es el resultado del valor intrínseco de la opción, es decir, la diferencia que existe entre el precio de mercado del activo subyacente y el precio de ejercicio de la opción.

$$
p=\max (0, K-S)
$$

$p$ : Precio de la opción

$K$ : Precio de ejercicio

$S$ : Precio Spot

En comparación con la compra directa del futuro de gas natural, la opción ofrece ventajas como apalancamiento adicional (la prima pagable es típicamente menor que el requisito de margen necesario para abrir una posición en los futuros de gas natural), la capacidad de limitar las posibles pérdidas ya que solo es la prima pagada para comprar la opción. En la práctica, NYMEX comercializa opciones sobre contratos de futuros de gas natural que se liquidan en entrega. ${ }^{9}$

Marginación del contrato de opciones.

Tal como en el caso de los futuros, la cámara de compensación debe asegurar que el riesgo que se corra sea el menor posible, por lo que en la transacción de opciones también se debe realizar la marginación. Los componentes de la marginación para este tipo de opciones son el margen por prima, margen por riesgo y margen por entrega que al igual que en los futuros, el margen por entrega sustituye al margen por riesgo si se trata de un tipo de entrega física.

El cálculo del margen por prima implica calcular la prima de mercado por el número de activos que ampara cada contrato multiplicado por la posición neta sobre el contrato de opción.

$$
C A P=\psi O
$$

CAP: Componente de margen por prima de la opción correspondiente al subyacente $\psi$ : Parámetro de margen por prima de la opción correspondiente al subyacente $O$ : Posición neta de la opción correspondiente al subyacente

\footnotetext{
${ }^{9}$ Véase al respecto Díaz-Tinoco y Venegas-Martínez (2004).
} 
Para calcular el margen por riesgo se utiliza simulación Monte Carlo con lo cual se simulan las alzas/bajas del precio del subyacente. Así se obtiene:

$$
C A R O=\left(\varphi^{z}-\psi\right) O, \text { donde: }
$$

CARO: Componente de margen por riesgo de la opción.

$\left(\varphi^{z}-\psi\right)$ : Es el parámetro de Mejor/Peor escenario de margen por riesgo de cada escenario simulado. En donde $z$ puede tomar valores enteros de 1 a 5 y desde -1 a -5 , en el caso del mejor o peor escenarios, respectivamente (Díaz-Tinoco y Venegas-Martínez, 2004).

$O$ : Posición neta de la opción correspondiente al subyacente

Finalmente, el margen total requerido para la opción, se calcula de la siguiente forma:

$$
A R O=C A P+C A R O
$$

$A R O$ : Margen requerido de la opción correspondiente

CAP: Componente de margen por prima de la opción

CARO: Componente de margen por riesgo de la opción

\subsection{Modelo binomial de valuación de opciones Cox, Ross y Rubinstein}

El modelo binomial Cox, Ross y Rubinstein (1979) proporciona una fórmula para valuar teóricamente una opción europea de compra sobre un título de capital que no paga dividendos. Su proceso resalta tres pasos fundamentales.

1. Calcular los probables precios futuros de los activos subyacentes al vencimiento.

2. Calcular el pago de la opción al vencimiento para cada uno de los precios subyacentes potenciales.

3. Descontar a valor presente los pagos para determinar el precio de la opción hoy.

Mediante el desarrollo de un árbol binomial se observa la posible trayectoria de los precios, tal que $c_{u}$ es el pago de la opción cuando hay un aumento en el precio del activo, mientras que $c_{d}$ muestra el pago de la opción cuando hay una disminución en el valor del activo.

$$
c_{u}=\max \left(u S_{t}-k_{t}, 0\right)
$$

$u S_{t}$ : Incremento en el precio spot del activo en el instante $t$ $k_{t}$ : Precio de la opción en el instante $t$

$$
c_{d}=\max \left(d S_{t}-k_{t}, 0\right)
$$

$u S_{t}$ : Disminución en el precio spot del activo en el instante $t$ $k_{t}$ : Precio de la opción en el instante $t$

Las ecuaciones del modelo son:

$$
\left[-\left(\frac{\left(c_{u}-c_{d}\right)}{S_{t}(u-d)}\right) d S_{t}+c_{d}\right] e^{r(T-t)}=-\left(\frac{c_{u}-c_{d}}{S_{t}(u-d)}\right) S_{t}+c_{t}
$$


Que lleva a

$$
c_{t}=\left[\left(\frac{e^{r(T-t)}-d}{u-d}\right) c_{u}+\left(\frac{d-e^{r(T-t)}}{u-d}\right) c_{d}\right] e^{r(T-t)}
$$

El valor del parámetro $p$ es: $p=\frac{e^{r(T-t)}-d}{u-d}$, obsérvese que se debe cumplir $u>d$, de donde:

$$
1-p=\frac{u-e^{r(T-t)}}{u-d}>\frac{d-e^{r(T-t)}}{u-d}=-p
$$

Con base en las ecuaciones de los parámetros $p, 1-p$ y $c_{t}$ ahora se busca el precio de la opción, lo cual se realiza descontando el nodo de vencimiento a valor presente,

$$
c_{t}=\left[p c_{u}+(1-p) c_{d}\right] e^{-r(T-t)} .
$$

\section{Cálculo del pronóstico de oferta y demanda de gas natural; simulación de precios de futuros y opciones}

\subsection{Estimación de la demanda de gas natural por el modelo de Grey}

Los datos de la demanda de gas natural, total y por sector, se encontraron en el Sistema de Información Energética para el periodo 1993 - 2017, los parámetros $\hat{a}$ y $\hat{b}$ se calcularon con el software R y son estadísticamente significativos en todos los casos. A continuación, en la tabla 1 se muestran los resultados del pronóstico de demanda de gas natural total y por sector mediante el modelo de Grey GM(1,1) desde 2011 hasta 2030, también se incluyen los datos reales hasta 2016. Como complemento, en las figuras 2 a 5 se presentan las gráficas de cada una de las variables de

\begin{tabular}{|c|c|c|c|c|c|c|c|c|c|c|c|c|c|c|c|c|c|c|c|}
\hline Año & 2012 & 2013 & 2014 & 2015 & 2016 & 2017 & 2018 & 2019 & 2020 & 2021 & 2022 & 2023 & 2024 & 2025 & 2026 & 2027 & 2028 & 2029 & 2030 \\
\hline Demanda Total & 6678 & 6952 & 7209 & 7504 & 7619 & & & & & & & & & & & & & & \\
\hline Grey GM(1,1) & 6323 & 6726 & 7169 & 7685 & 8250 & 9059 & 8803 & 9194 & 9603 & 10030 & 10477 & 10943 & 11430 & 11938 & 12469 & 13024 & 13604 & 14209 & 14841 \\
\hline $\begin{array}{l}\text { Demanda } \\
\text { eléctrica }\end{array}$ & 3111 & 3323 & 3500 & 3798 & 3878 & & & & & & & & & & & & & & \\
\hline Grey GM(1,1) & 4774 & 5276 & 5851 & 6555 & 7291 & 8301 & 5273 & 5687 & 6133 & 6615 & 7134 & 7694 & 8298 & 8949 & 9651 & 10409 & 11226 & 12107 & 13057 \\
\hline $\begin{array}{l}\text { Demanda } \\
\text { Petrolera }\end{array}$ & 2273 & 2272 & 2276 & 2200 & 2122 & & & & & & & & & & & & & & \\
\hline Grey GM(1,1) & 1873 & 1863 & 1895 & 1967 & 2158 & 2471 & 2480 & 2526 & 2573 & 2620 & 2669 & 2718 & 2768 & 2820 & 2872 & 2925 & 2979 & 3034 & 3090 \\
\hline $\begin{array}{l}\text { Demanda } \\
\text { Industrial }\end{array}$ & 1181 & 1240 & 1313 & 1376 & 1484 & & & & & & & & & & & & & & \\
\hline Grey GM(1,1) & 1462 & 1511 & 1531 & 1508 & 1453 & 1323 & 1386 & 1420 & 1454 & 1489 & 1525 & 1562 & 1600 & 1639 & 1678 & 1719 & 1761 & 1803 & 1847 \\
\hline
\end{tabular}
contenidas en esta tabla.

Tabla 1. Demanda real y estimada de gas natural (MMPCD)

Fuente: Prospectiva del Gas Natural 2017 - 2031 (SENER, 2017) 


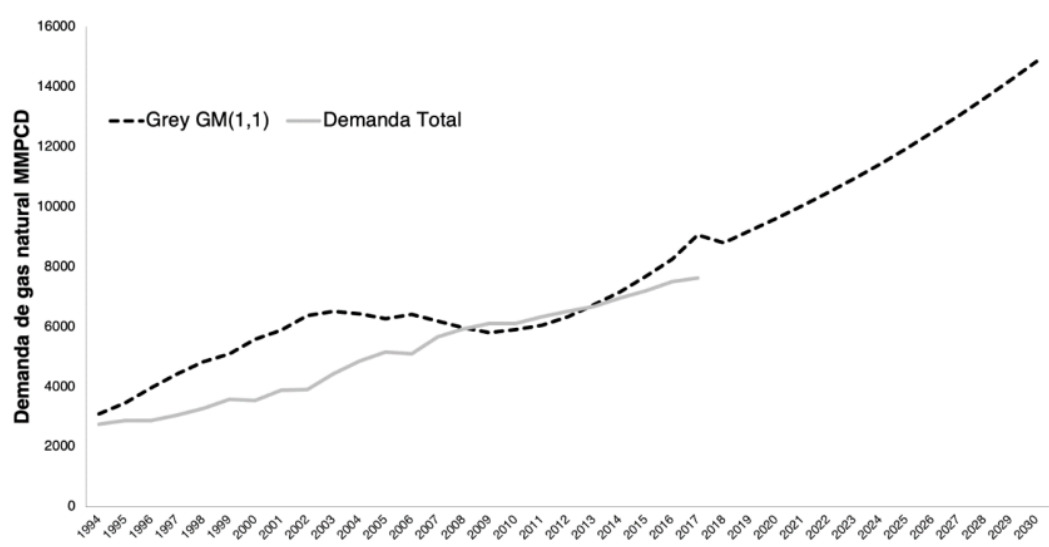

Figura 2. Demanda total de gas natural

Fuente: Elaboración propia con datos del SIE

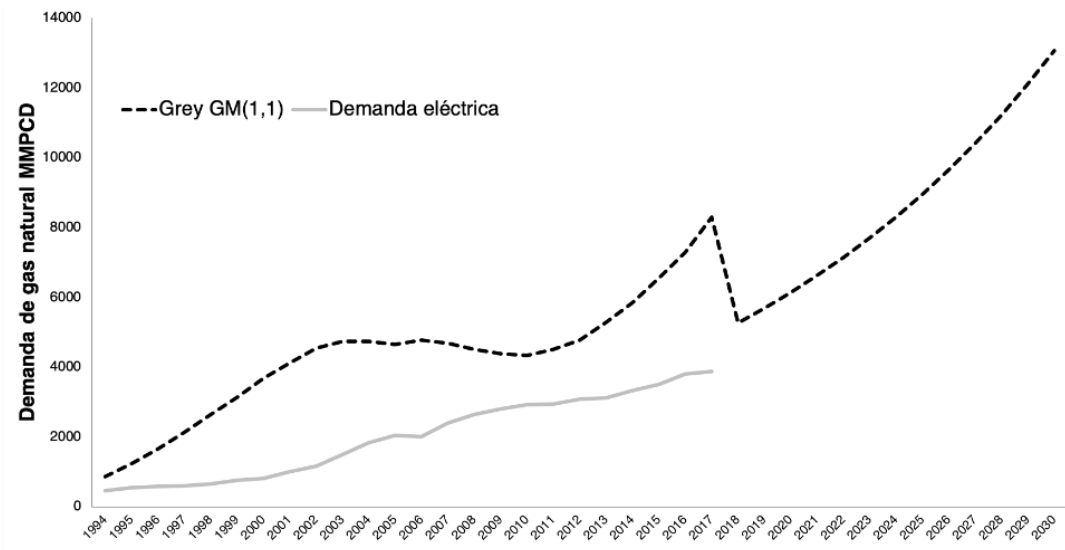

Figura 3. Demanda de gas natural del sector eléctrico Fuente: Elaboración propia con datos del SIE

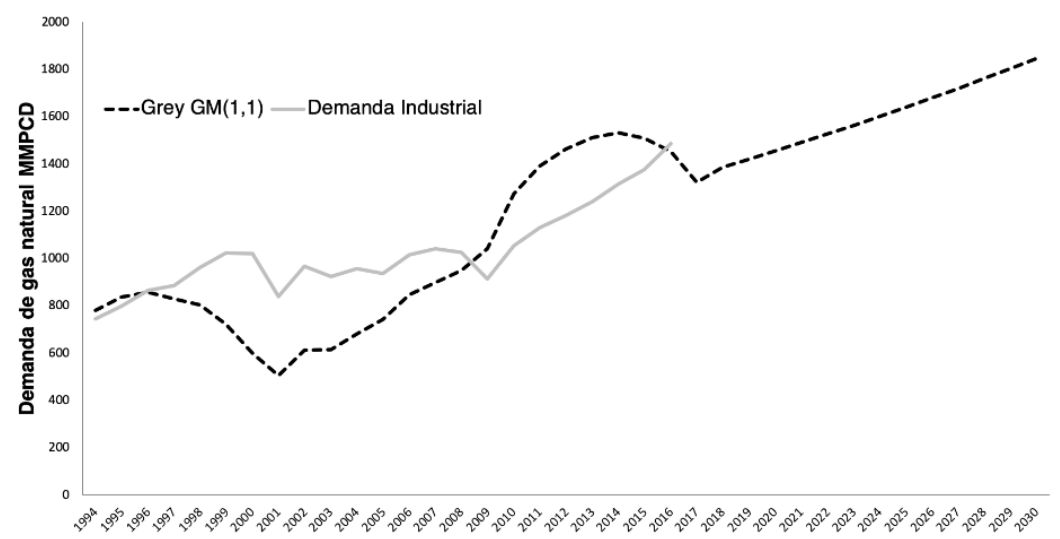

Figura 4. Demanda de gas natural del sector industrial Fuente: Elaboración propia con datos del SIE 


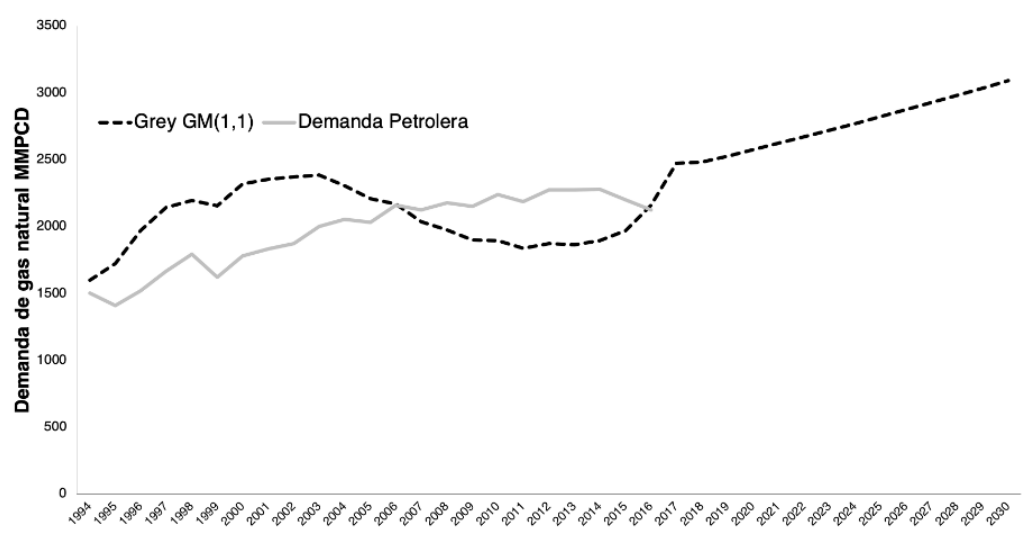

Figura 5. Demanda de gas natural del sector petrolero Fuente: Elaboración propia con datos del SIE

De acuerdo a los resultados obtenidos mediante el modelo de Grey GM(1,1), se infiere que la demanda total y por sector se incrementarán año con año.

El modelo de Grey GM(1,1), muestra tendencias positivas de consumo de gas natural a través del tiempo, también destaca que al ser descrita por una ecuación diferencial de una variable, la dinámica de dicha variable es más fácil de percibir, incluso, Xie (2009), Xie y Li (2009), Ma y Wu (2009), Ma y Li (2010) confirman que debido a que el consumo de gas natural se ve afectado por muchos factores (algunos claros y otros no), de alto grado de complejidad y de carácter no lineal; el resultado obtenido mediante el modelo de Grey GM(1,1) es simple y preciso, de tal modo que se ajusta razonablemente bien a la proyección de los datos.

Tal y como se puede apreciar en los gráficos anteriores, el modelo de Grey, aunque eficiente en el largo plazo, es incapaz de ajustar los cambios de corto plazo, producto de la pléyade de factores que afectan la demanda, esta debilidad es acometida por nuestra propuesta de utilizar de manera complementaria un modelo de reversión a la media.

\subsection{Estimación de la demanda de gas natural por el modelo de reversión a la media}

Basados en el modelo de reversión a la media de Vasicek (1977), se presentan los resultados promedio de un modelo Monte Carlo ajustado a los puntos de demanda históricos de gas natural, generando tasas de crecimiento de la demanda de gas más cercanas a los datos reales.

El modelo de reversión a la media es una alternativa que permite calcular el pronóstico de la demanda de gas natural, se consideran los datos de consumo del periodo 1993 - 2017, en primer lugar, se transforma la base de datos de demanda de gas natural en tasas de crecimiento. Después, se desarrolló un modelo de regresión lineal simple con un proceso autorregresivo de orden 1 con tendencia a través del software R. El parámetro $(a)$ velocidad de reversión a la media y el parámetro (b) media de largo plazo, se obtienen por medio de la sustitución de los estimadores $\beta_{0}=a b$ y $\beta_{1}=$ $1-a$

$$
r_{t}=\beta_{0}+\beta_{1} r_{t-1}+\sigma d Z_{t}
$$


La media incondicional de $r_{t}$ es

$E\left[r_{t}\right]=\frac{\beta_{0}}{\left(1-\beta_{1}\right)}=\frac{a b}{(1-1+a)}=\frac{a b}{a}=b, \quad$ y su varianza incondicional está dada por:

$$
\operatorname{Var}\left[r_{t}\right]=\frac{\sigma^{2}}{1-\beta_{1}^{2}}=\frac{\sigma^{2}}{\left[1-(1-a)^{2}\right]}
$$

En la sección 3.2 se planteó que $r_{t}$ es estimada mediante la siguiente ecuación

$$
\hat{r}(t)=b+\left(r_{0}-b\right) e^{-a t}+\frac{\sigma e^{-a t} \sqrt{e^{2 a t}-1}}{\sqrt{2 a}} Z
$$

En las figuras 6 a 9 se muestran las tasas de crecimiento de la demanda total de gas natural, obtenidas mediante el modelo combinado de "Grey-Vasicek" propuesto en el artículo, para el sector eléctrico, para sector el industrial y para la demanda del sector petrolero, respectivamente.

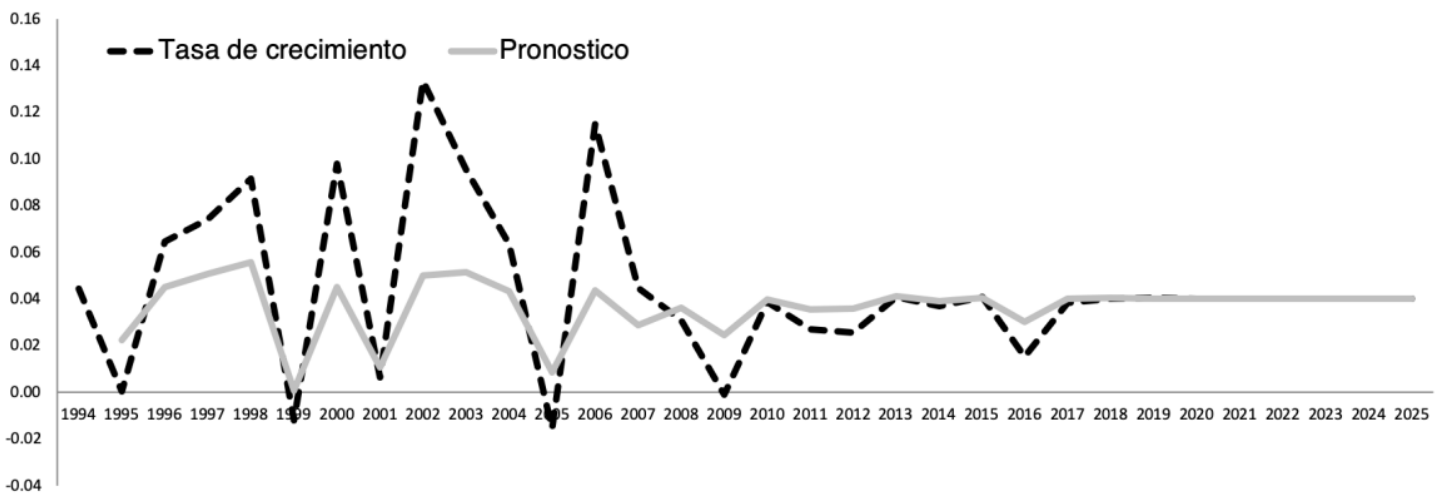

Figura 6. Tasa de crecimiento de la demanda total de gas natural Fuente: Elaboración propia con datos del SIE

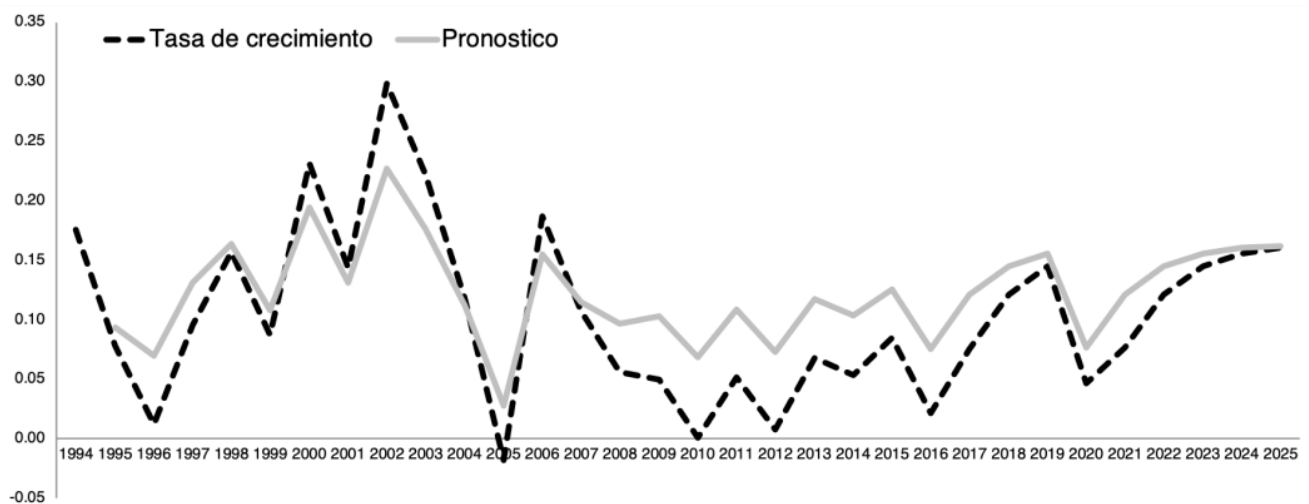

Figura 7. Tasa de crecimiento de la demanda del sector eléctrico

Fuente: Elaboración propia con datos del SIE 


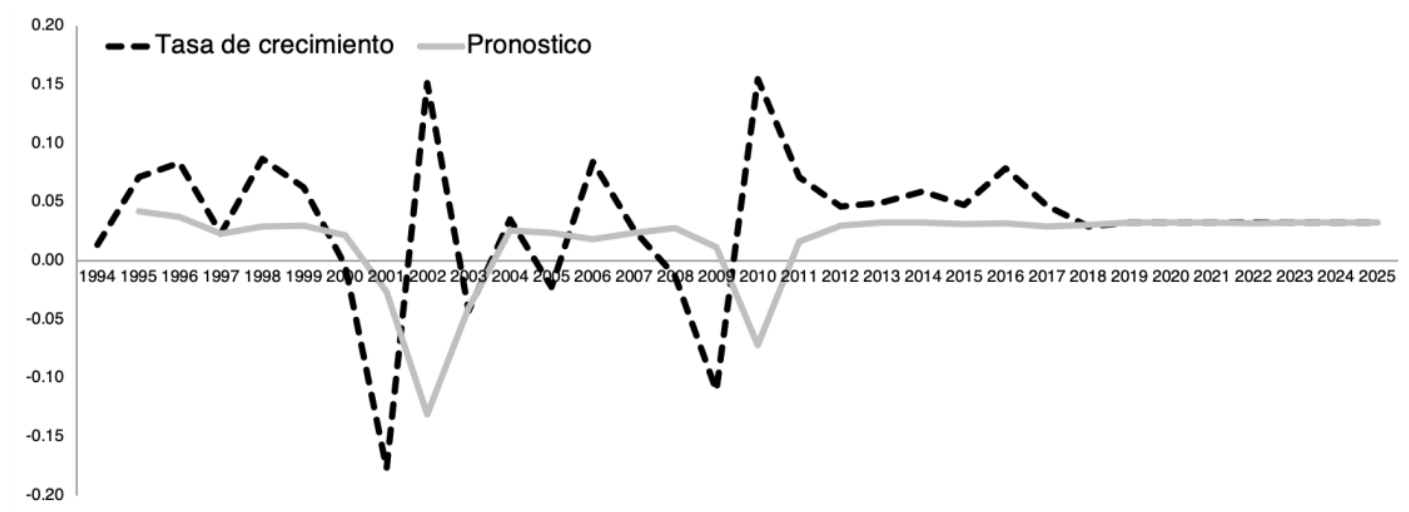

Figura 8. Tasa de crecimiento de la demanda del sector industrial Fuente: Elaboración propia con datos del SIE

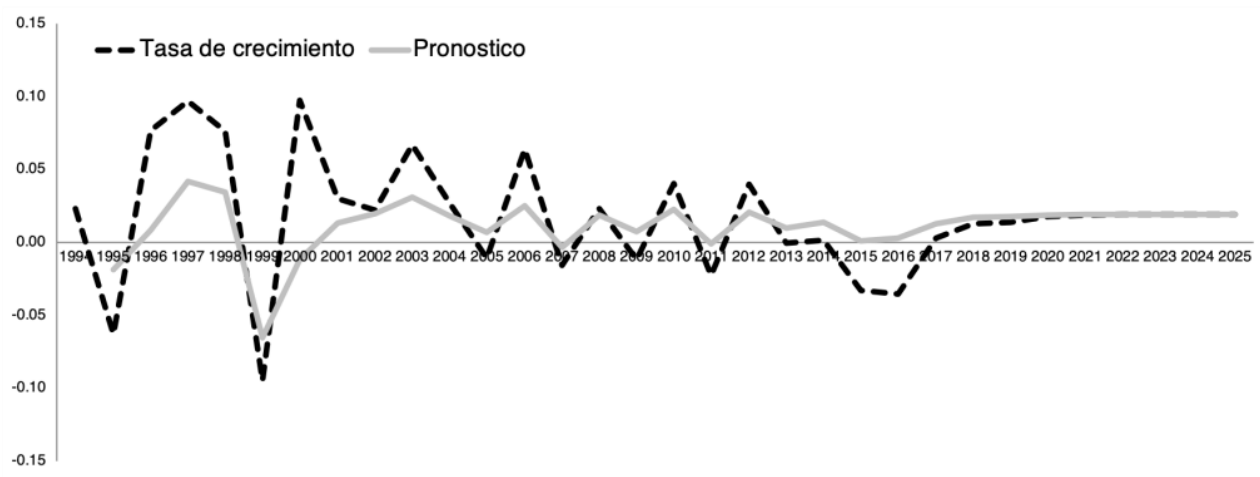

Figura 9. Tasa de crecimiento de la demanda del sector petrolero Fuente: Elaboración propia con datos del SIE

Con base en los resultados obtenidos, se observa que las tasas de crecimiento son estables y las variables se ajustan rápidamente a su media de largo plazo. Para la demanda total, la velocidad de reversión a la media es 1.2296 y la tasa de crecimiento promedio hacia 2025 será de $4.02 \%$, en cuanto a la tasa de crecimiento promedio por sector se observa que la tasa del sector eléctrico será de $15.2 \%$, su velocidad de reversión a la media será de 0.6509 , mientras que el crecimiento del sector industrial y petrolero será más discreto, 3.45 \% y 1.09 \% con velocidad de reversión a la media de 1.2622 y 1.2245 respectivamente. Se observa que las tasas de crecimiento de la demanda general de gas natural son positivas, por lo que la se pronostica que la demanda crecerá.

El crecimiento de la demanda del sector eléctrico presiona la capacidad para generar electricidad con gas natural, según (SENER, 2017), el sector público será el que demandará la mayor cantidad. En concreto, la Comisión Federal de Electricidad (CFE) lo utilizará para la generación eléctrica a través de sus plantas de ciclo combinado que presentan un uso eficiente y de menor costo de operación. 
En el sector industrial se pronostica el crecimiento de la demanda de gas natural. Se estima que la incorporación de nuevos gasoductos y permisos de distribución por medio de ductos, cubrirán a usuarios industriales, factor suficiente para que se incremente la demanda. Y el sector petrolero presentará una tendencia decreciente de consumo si continúa su decaimiento.

\subsection{Estimación del modelo de Hubbert}

Se utilizó el modelo de Hubbert para la estimación de la producción de gas natural en México, debido a que es adecuado usar este modelo en países tomadores de precios en el recurso analizado (Szklo et al. 2007). Para desarrollar el modelo de Hubbert se tomaron los datos de los anuarios estadísticos de Pemex (1977), Pemex (1990), Pemex (1999) y del Balance de Gas Natural. El periodo de análisis va de 1938 a 2017. El ritmo de extracción y la cantidad total de gas natural disponible se obtienen por el método de MCO.

Los resultados son: El ritmo de extracción $a=0.1233$, la cantidad total de gas natural disponible $Q=a / b=103$. En cuanto al pico de Hubbert, éste se alcanzó en el año 2009, el decaimiento de la producción de gas natural será en 2040, resultados consistente con las proyecciones hechas por (SENER, 2017), respecto a la producción de gas natural. En la figura 10 se muestra el resultado de la linealización de Hubbert. En la figura 11 se presenta la curva de Hubbert utilizada para proyectar la producción histórica de gas natural.

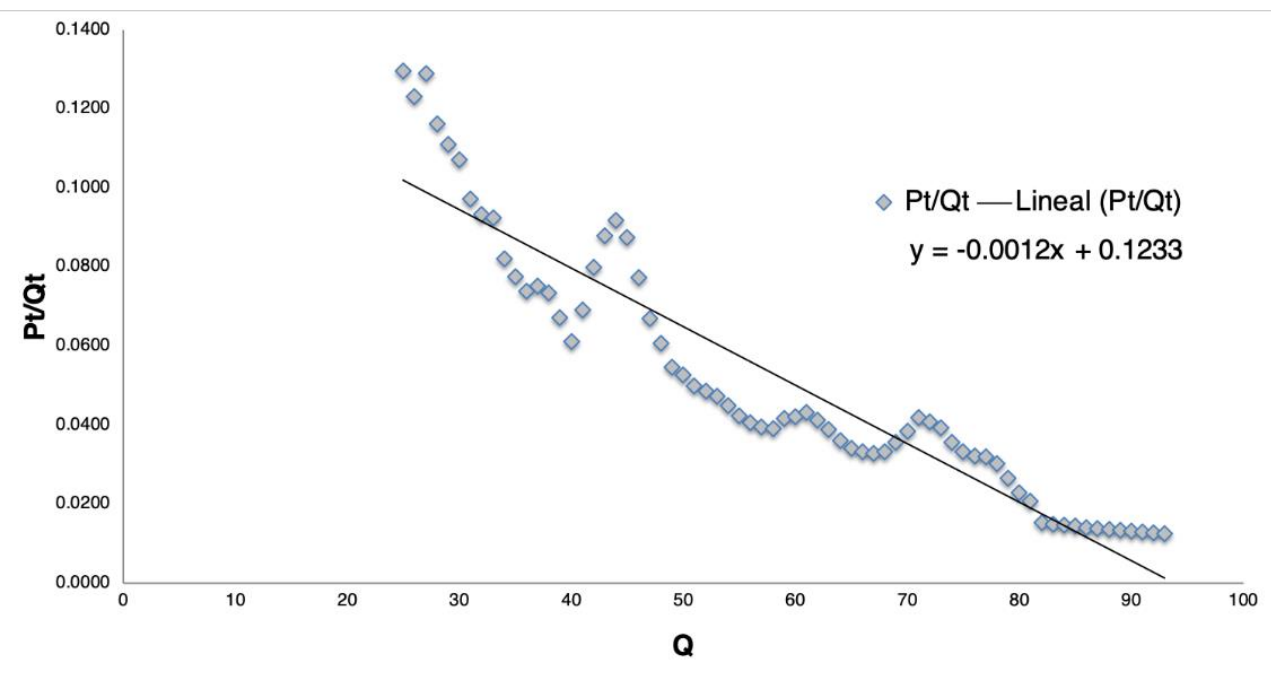

Figura 10. Linealización de Hubbert

Fuente: Elaboración con datos del anuario estadístico de Pemex y Balance de Gas Natural 


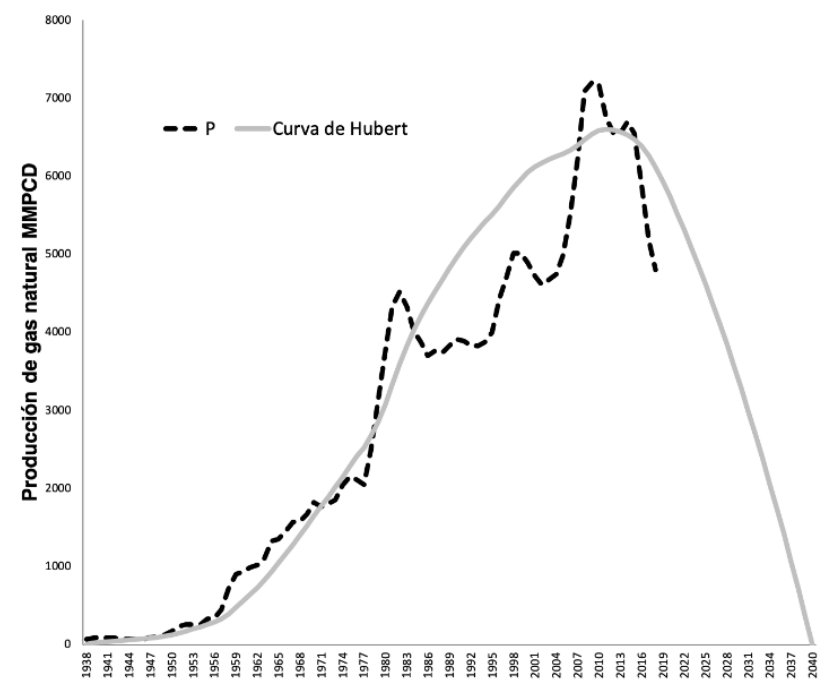

Figura 11. Producción histórica y curva de Hubbert

Fuente: Elaboración con datos del anuario estadístico de Pemex y Balance de Gas Natural (CNH)

En la tabla 2 se presentan los resultados de la proyección de producción de gas natural obtenidos mediante el modelo de Hubbert.

Tabla 2. Estimación con modelo de Hubbert

\begin{tabular}{|l|c|c|c|c|c|c|c|c|c|c|c|c|c|}
\hline & $\mathbf{2 0 1 8}$ & $\mathbf{2 0 1 9}$ & $\mathbf{2 0 2 0}$ & $\mathbf{2 0 2 1}$ & $\mathbf{2 0 2 2}$ & $\mathbf{2 0 2 3}$ & $\mathbf{2 0 2 4}$ & $\mathbf{2 0 2 5}$ & $\mathbf{2 0 2 6}$ & $\mathbf{2 0 2 7}$ & $\mathbf{2 0 2 8}$ & $\mathbf{2 0 2 9}$ & $\mathbf{2 0 3 0}$ \\
\hline $\begin{array}{l}\text { Modelo de Hubbert } \\
\text { MMPCD }\end{array}$ & 6118 & 5930 & 5733 & 5527 & 5312 & 5089 & 4856 & 4615 & 4366 & 4108 & 3841 & 3566 & 3282 \\
\hline
\end{tabular}

Fuente: Elaboración con datos del anuario estadístico de Pemex y Balance de Gas Natural (CNH)

\subsection{Balance del mercado de gas natural}

Realizado el pronóstico de oferta y demanda de gas natural, se contrastan ambos resultados para identificar si existe capacidad de abastecer el consumo por medio de la producción nacional, esto se describe gráficamente en la figura 12 .

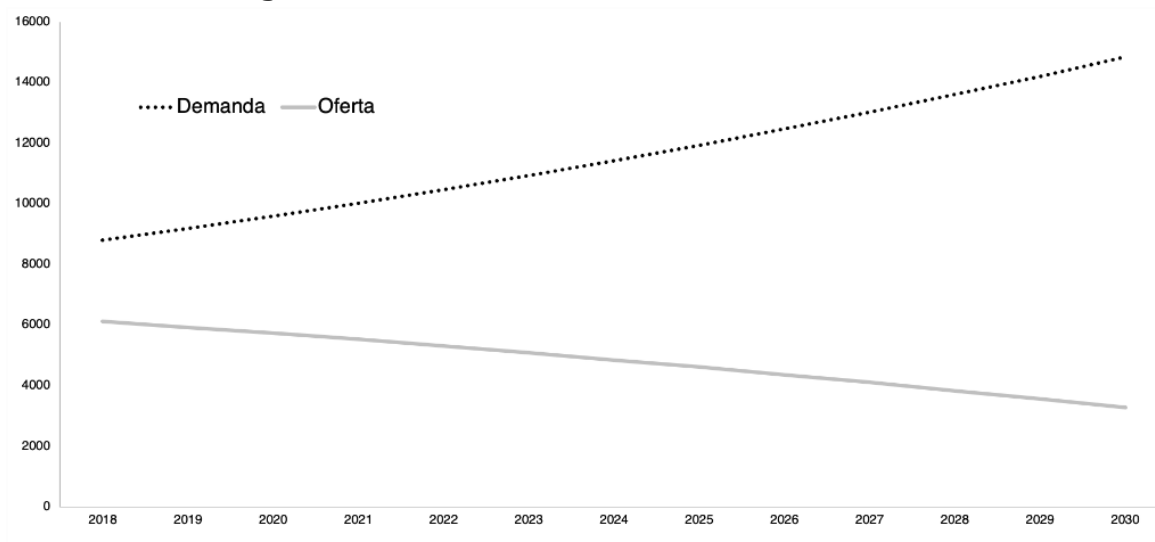

Figura 12. Oferta y demanda de gas natural MMPCD

Fuente: SIE (Sistema de Información Energética) 
Como se puede apreciar, existe una brecha entre la producción y el consumo pronosticado de gas natural, por lo que la demanda no será compensada con la producción nacional. Conviene mencionar que en México, actualmente (de manera oficial) no hay en operación comercial proyectos de extracción de hidrocarburos por fuentes no convencionales como el gas de lutitas, a pesar de que se han realizado operaciones de exploración de campos en Burgos autorizados por la CNH. La explotación de dichos campos permitiría cerrar la brecha según CNH (2018). En comparación al mercado internacional, donde se permite la extracción de gas natural por medio de fuentes no convencionales, lo que les da la oportunidad de producir más y exportar el remanente de su oferta ofreciendo precios bajos. En modo estricto, la limitada capacidad productiva no haría factible la comercialización con contratos de derivados sobre gas natural; sin embargo, se realizará una simulación de manera teórica.

\subsection{Simulación del contrato de futuros de gas natural en México}

Para la negociación de contratos de futuros, es primordial la conveniencia de un punto de entrega que ofrezca una buena ubicación geográfica y logística, por lo que se eligión la ciudad de Reynosa (Tamaulipas), la cual cumple con las características necesarias. Se consideró como referencia técnica las especificaciones del nodo Henry Hub (CME, G., 2018).

\begin{tabular}{|l|l|}
\hline Demanda & $\begin{array}{l}\text { Tamaulipas se ubica dentro de la región noreste junto con Coahuila, Chihuahua, Durango } \\
\text { y Nuevo León. En la región existe una demanda dinámica, la cual tiene una participación } \\
\text { de } 32.8 \% \text { (SENER, 2017) }\end{array}$ \\
\hline Producción & $\begin{array}{l}\text { La región tiene a su alcance la exploración y producción en tierra y en mar. Según SENER } \\
\text { (2016), las regiones norte y sur, en promedio extraen un 65\% a nivel nacional. }\end{array}$ \\
\hline Comercio & $\begin{array}{l}\text { La región presenta considerables volúmenes de importación de gas natural debido a la } \\
\text { conexión de ductos con la frontera sur de los Estados Unidos y la entrada de GNL por barco } \\
\text { en la terminal de regasificación de Altamira, Tamaulipas. Además, en esta región se } \\
\text { concentra el mayor número de zonas geográficas de distribución del país (SENER, 2017). }\end{array}$ \\
\hline
\end{tabular}

La figura 13 nos muestra la distribución geográfica de la infraestructura de ductos de gas natural de México.

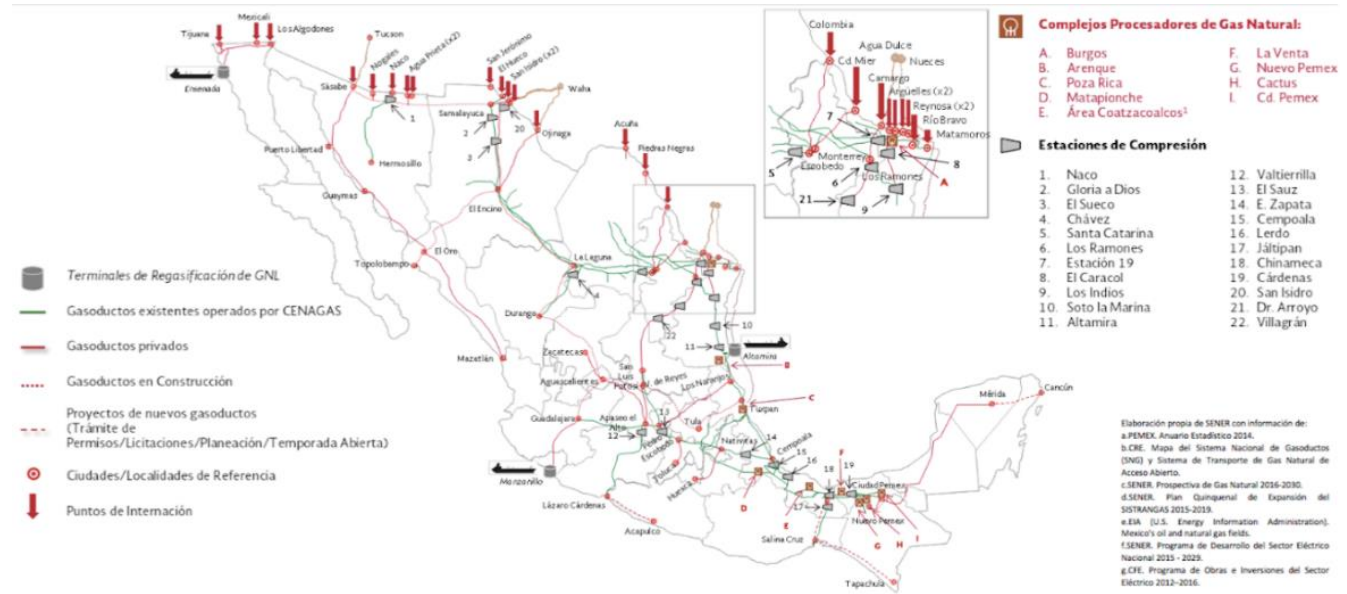

Figura 13. Infraestructura nacional del gas natural (2018)

Fuente: SENER (2018). Política Pública en materia de almacenamiento de gas natural 


\section{Estructura del contrato de futuros:}

El contrato se simula de acuerdo a las disposiciones que operan en MexDer. A continuación, se muestran las características principales de la propuesta de contrato de futuros con activo subyacente gas natural.

\begin{tabular}{|l|l|}
\hline $\begin{array}{l}\text { Activo subyacente } \\
\text { Número de unidades del activo } \\
\text { subyacente o valor nominal que } \\
\text { ampara un contrato de futuro }\end{array}$ & El activo subyacente es gas natural. \\
\hline Unidad de cotización & $\begin{array}{l}\text { La celebración del contrato se cotizará en pesos mexicanos por giga joule } \\
\text { del precio futuro de gas natural. }\end{array}$ \\
\hline Procedimiento de liquidación & Entrega física en Reynosa, Tamaulipas (México). \\
\hline Precio de liquidación al vencimiento do futuro ampara 10,000 Giga Joules (GJ) \\
$\begin{array}{l}\text { El precio de liquidación al vencimiento para el contrato de futuro será lo } \\
\text { que resulte de obtener el precio spot a través del índice de referencia } \\
\text { nacional de precios de gas natural al mayoreo (IPGN) en pesos por giga } \\
\text { joule multiplicado por una tasa continuamente capitalizable del costo de } \\
\text { almacenamiento como porcentaje del precio del gas natural más la tasa } \\
\text { de referencia (TIIE) a 28 días publicada por Banco de México menos la } \\
\text { tasa de conveniencia, según lo estipulado en la metodología de valuación } \\
\text { de MexDer. }\end{array}$ \\
\hline
\end{tabular}

\section{Ejemplo de un contrato de futuros}

En primer lugar, se simulan los parámetros de marginación, se supone que en el mercado existen 20 posiciones cortas de GNMX JL18 y 10 posiciones largas de GNMX JL18, además un factor de determinación de 0.50. Se calcula la vme a través del método VaR por simulación Montecarlo con 10,000 iteraciones en el software $\mathrm{R}$, se consideran como datos iniciales el precio promedio mensual del IPGN (CRE, 2018) desde julio de 2017 hasta agosto de 2018, de la misma fuente se extrae la volatilidad, además se supone un alpha igual a 0.01 .

Tabla 3. Elementos del contrato de futuros sobre el precio de gas natural.

\begin{tabular}{|l|l|l|l|l|l|l|}
\hline Contrato & Posición & VME & Opuesto & Entrega & Tamaño de contrato & Posición \\
\hline GNMX JL18 & Larga & 0.13 & 650 & 6500 & 10,000 & 10 \\
\hline GNMX JL18 & Corta & 0.13 & 650 & 6500 & 10,000 & 20 \\
\hline
\end{tabular}

La AIM del GNMX es igual a la suma del margen por entrega y margen por posiciones opuestas, sustituyendo los datos en las ecuaciones (12) y (13), el valor obtenido es de \$71,500, lo cual cubrirá la pérdida esperada por cada contrato vigente en la Cámara de Compensación.

Se retoman los datos de la tabla 3 para obtener la marginación, donde se supone que el portafolio de un distribuidor de gas natural está compuesto por 20 posiciones cortas de GNMX JL18 y 10 posiciones largas de GNMX JL18 para entregar en 7 días, la AIM es \$71,500, el precio spot es de \$74.154 (el precio spot y su rendimiento se simuló por el método Montecarlo con 1,000 iteraciones mediante el software $\mathrm{R}$, véase apéndice), se utiliza la ecuación (11) para hacer los cálculos de la tabla 4 con los siguientes datos: la tasa TIIE a 28 días es de $8.12 \%$, el costo de almacenamiento es de $5 \%$ sobre el valor del activo subyacente y la tasa de conveniencia es del $4.5 \%$, obteniéndose los siguientes valores: 
Tabla 4. Resultados del contrato de futuros sobre el precio de gas natural.

\begin{tabular}{|l|l|l|l|l|l|l|}
\hline & Precio Spot & Precio del Futuro & AIM & Flujo & Aportación Margen & Saldo final \\
\hline 7 & 73.2560 & 73.3788 & 71500 & & $<->$ & 71500 \\
\hline 6 & 73.3401 & 73.4455 & 71500 & 6669 & $<->$ & 78169 \\
\hline 5 & 73.3561 & 73.4439 & 78169 & 6510 & $<->$ & 84679 \\
\hline 4 & 73.2809 & 73.3511 & 84679 & -2773 & $<->$ & 81906 \\
\hline 3 & 73.3009 & 73.3536 & 81906 & -2528 & $<->$ & 79378 \\
\hline 2 & 73.3336 & 73.3687 & 79378 & -1013 & $<->$ & 78365 \\
\hline 1 & 73.3470 & 73.3646 & 78365 & -1426 & $<->$ & 76938 \\
\hline 0 & 73.3616 & 73.3616 & 76938 & -1727 & $<->$ & 75211 \\
\hline
\end{tabular}

\subsection{Simulación del contrato de opciones de gas natural en México}

Especificaciones del contrato de opciones sobre el contrato de futuro de gas natural (GNMX)

\begin{tabular}{|c|c|}
\hline Activo subyacente & $\begin{array}{l}\text { El activo subyacente es un contrato de futuro sobre gas natural. Al vencimiento } \\
\text { de una opción de compra, el valor será la diferencia entre el precio de } \\
\text { liquidación del futuro y el precio de ejercicio multiplicado por 10,000 GJ, o } \\
\text { cero, el que sea mayor. En el ejercicio de una opción de venta, el valor será la } \\
\text { diferencia entre el precio de ejercicio y el precio de liquidación del futuro de } \\
\text { GNMX multiplicado por } 10,000 \text { GJ, o cero, lo que sea mayor. }\end{array}$ \\
\hline Unidad de cotización & $\begin{array}{l}\text { La celebración del contrato se cotizará en pesos mexicanos por cada contrato } \\
\text { amparado de futuro del GNMX }\end{array}$ \\
\hline $\begin{array}{l}\text { Número de unidades del activo } \\
\text { subyacente o valor nominal } \\
\text { que ampara el contrato de } \\
\text { opción }\end{array}$ & \\
\hline Tipos de contratos de opción & $\begin{array}{l}\text { En todo momento MexDer mantendrá la posibilidad de cotizar contratos de } \\
\text { opción de compra (Call) y de venta }(P u t) \text {. }\end{array}$ \\
\hline Estilo del contrato de opción & Europeo. Se ejercen en la fecha de vencimiento. \\
\hline Series & $\begin{array}{l}\text { MexDer listará y mantendrá disponibles para su negociación contratos de } \\
\text { opción sobre el futuro del GNMX, tanto de compra como de venta en los precios } \\
\text { de ejercicio y sobre una base mensual, lo que significa que habrá contratos } \\
\text { para el año en curso y los próximos } 12 \text { meses del calendario. Los contratos } \\
\text { mensuales para un nuevo año del calendario se agregarán después de la } \\
\text { terminación de la negociación en el contrato de diciembre del año en curso. }\end{array}$ \\
\hline Método de liquidación & Financiera \\
\hline
\end{tabular}

Ejemplo del contrato de opciones cuyo valor es calculado mediante el método Cox, Ross y Rubinstein.

A continuación, se procede a realizar la valoración de la prima del contrato de opción, es decir, determinar un precio "justo" por el derecho a comprar o vender el futuro de gas natural a un precio pactado en una fecha preestablecida. 
Se retoman los datos utilizados para el contrato de futuros sobre gas natural con precio spot igual a 74.154, precio de ejercicio es 73.8798, el periodo es a 7 días, la TIIE $8.12 \%$ y una volatilidad de 0.05747 , se emplea la paquetería CRR Binomial Tree Option del software R para obtener el precio teórico de una opción call y una opción put, mediante el modelo de Cox, Ross y Rubinstein (véase apéndice), así como la representación gráfica del árbol binomial para cada caso.

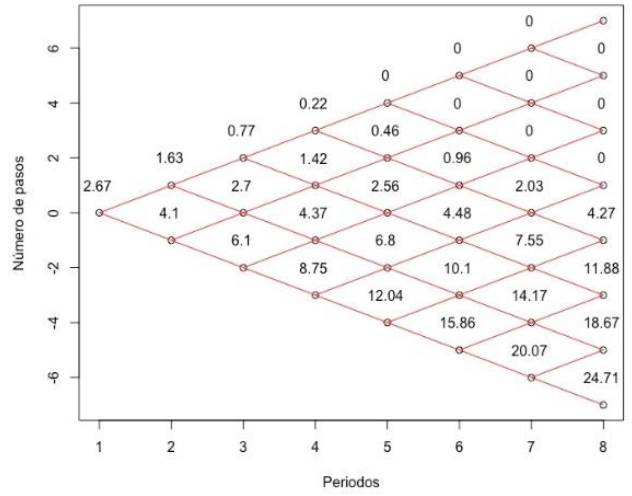

Figura 14. Árbol binomial de la opción call (\$) Fuente: Elaboración propia con software R

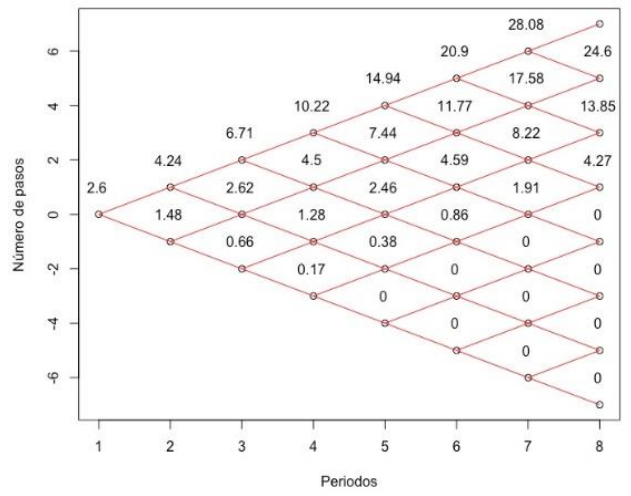

Figura 15. Árbol binomial de la opción put (\$) Fuente: Elaboración propia con software R

\section{Simulación de la marginación del contrato de opciones}

Con base en la descripción realizada de la marginación para contratos de opciones, se procede a realizar la simulación de marginación para un portafolio de grupo clase conformado por dos contratos de opciones, se supone que existen 20 posiciones call y 40 posiciones put, cada opción ampara un contrato de futuro de gas natural, la prima de mercado se calculó usando el método Cox, Ross y Rubinstein con los datos previamente usados para el caso de los futuros, las primas se pagan en pesos.

Tabla 5. Elementos del contrato de opciones europeas sobre el precio de gas natural.

\begin{tabular}{|l|l|l|l|l|l|}
\hline Contrato & $\begin{array}{l}\text { Tipo de } \\
\text { Posición }\end{array}$ & $\begin{array}{l}\text { Prima de } \\
\text { Mercado }\end{array}$ & AIM por Prima & $\begin{array}{l}\text { Tamaño } \\
\text { Contrato }\end{array}$ & Posición \\
\hline OGNMX JL18 Call & Larga & 2.709474 & -54.18948 & 1 & -20 \\
\hline OGNMX JL18 Put & Corta & 2.554401 & 102.17604 & 1 & 40 \\
\hline
\end{tabular}

Finalmente, el requerimiento de margen total para el portafolio será igual a la suma de los componentes de margen por riesgo y margen por prima.

Tabla 6. Resultados del contrato de opciones europeas sobre el precio de gas natural,

\begin{tabular}{|c|c|c|}
\hline \multicolumn{3}{|c|}{ Margen total } \\
\hline Margen por riesgo & Margen por prima & Margen total \\
\hline 22.81344 & 47.98656 & 70.8 \\
\hline
\end{tabular}


Como puede apreciarse desde el punto de vista financiero ambos contratos de derivados son adecuados, sin embargo, el bajo volumen de producción de gas natural en México pone en riesgo la viabilidad operativa de estos, dado que esto implicaría un bajo volumen de operaciones de cobertura.

\section{Conclusiones}

Respecto al resultado obtenido sobre el pronóstico de demanda de gas natural por el modelo de Grey GM $(1,1)$ - Vasicek, se infiere que el consumo se expandirá de manera general y en cada uno de los sectores de estudio; en contraste, los resultados obtenidos de la producción nacional a través del modelo de Hubbert, se estima que la producción nacional disminuirá. El balance de oferta y demanda indica que en el futuro la brecha entre consumo y producción se incrementará, favoreciendo al consumo (demanda).

La transacción de gas natural a través de contratos de derivados requiere un mercado líquido tal que la oferta y la demanda coexistan hipotéticamente cerca de un equilibrio dinámico, por lo que el uso de contratos de derivados permitiría asegurarse de este objetivo, así mismo, permitiría la disminución de riesgos ante una oscilación de precios cuando dicho equilibrio no se cumpliera; sin embargo, con base en los datos empíricos, la actual plataforma de producción es pequeña en comparación con la demanda, por lo cual, con la actual producción nacional de gas natural no se tiene la capacidad de poder garantizar la operación de contratos de derivados a los consumidores, debido a su propia restricción de producción. Además, hay que señalar que el consumidor puede importar gas natural en el mercado exterior, que satisfaga sus necesidades, incluso a un precio más bajo.

Es importante mencionar el hecho de que, aunque en el país exista un número considerable de cuencas para la potencial extracción de hidrocarburos, no significa que se tenga aprovechamiento pleno de ello. Uno de los objetivos de la reforma energética fue impulsar la inversión en la plataforma productiva y comercial de modo que el mercado de energéticos fuera rentable, tanto para los productores como para los consumidores, sin embargo, las condiciones externas al mercado nacional vuelven apremiante la atracción de inversión privada, por ejemplo, la extracción de hidrocarburos por métodos no convencionales en los Estados Unidos ha impulsado un incremento en la producción, dando como resultado precios bajos, e indirectamente a causando la importación de gas natural por parte de México mediante gasoductos conectados en la frontera de ambos países, lo cual provoca día con día, mayor dependencia energética y esto puede generar que en el futuro un problema de seguridad nacional.

Se puede inferir de acuerdo a los resultados, que en el mercado nacional de gas natural no existen ventajas competitivas tal y como se perciben en el mercado internacional, entendiéndose como una habilidad o conocimiento que incremente su eficiencia y le permita ser más competitivo. El origen de dichas ventajas, se puede dar de acuerdo a factores como el precio, tecnología o bajos costos en la producción.

En este sentido, García et al., (2018) sugiere que la producción está correlacionada con la tasa de rentabilidad, en lugar de la disponibilidad del recurso como se menciona en el modelo de Hubbert, por tanto, el pico Hubbert puede retrasarse si existe un nivel de precios del cual se obtenga una tasa de rentabilidad positiva. Tal y como se interpreta en (García et al., 2018), SENER ha puntualizado que primero se sustraen aquellos recursos que es posible obtener de forma más competitiva en relación 
a los costos, por lo que la disminución de la calidad del recurso tiende a aumentar el costo de extracción y disminuir los incentivos para la extracción. Por lo cual se infiere que un avance en la inversión de tecnología permite un uso más eficiente de la extracción al disminuir su costo.

Una línea de investigación complementaria a este trabajo podría ser calcular el valor mínimo y el óptimo de la extracción de gas natural para el uso de contratos de derivados de gas natural en el MexDer.

\section{Referencias}

[1] Amell, A., Agudelo, J., and Cadavid, F. (2016). El gas natural: ¿nuevo vector energético? Revista Facultad de Ingeniería, (25):36-48. DOI: https://doi.org/10.1016/j.jngse.2016.03.039

[2] Bere, N. P. F. (2010). A critical review of the peak oil phenomenon. Master's thesis, Norwegian School of Economics.

[3] CRE (2018). Índices de Referencia de Precios de Gas Natural. http://www.cre.gob.mx/IPGN/

[4] CME, G. (2018). Natural gas contract specs. https://www.cmegroup.com/trading/energy/naturalgas/naturalgascontractSpecsoptions.html.optionProductId=1352optionProductId=1352

[5] CNH (2018). Balance de gas natural. https://portal.cnih.cnh.gob.mx/estadisticas.php.

[6] Cox, J. C., Ross, S. A., and Rubinstein, M. (1979). Option pricing: A simplified approach. Journal of financial Economics, 7(3):229-263. DOI: https://doi.org/10.1016/0304-405x(79)90015-1

[7] Díaz-Tinoco, J. y F. Venegas-Martínez (2004). Márgenes con spread intraclase para el mercado mexicano de derivados. El Trimestre Económico, 71(3): 681-716.

[8] Frauendorfer, K. (2007). Derivatives in the gas industry: Valuation of natural gas storage facilities. Master's Thesis. University of St. Gallen.

[9] Hsu, C.-C. and Chen, C.-Y. (2003). Applications of improved grey prediction model for power demand forecasting. Energy Conversion and management, 44(14):2241-2249. DOI: https://doi.org/10.1016/s0196-8904(02)00248-0

[10] Hubbert, M. K. et al. (1956). Nuclear energy and the fossil fuel. In Drilling and production practice. American Petroleum Institute. DOI: https://doi.org/10.1016/0043-1648(58)90046-2

[11] Ibañez, F., R. Romero-Meza, S. Coronado-Ramírez y F. Venegas-Martínez (2016). Innovaciones financieras en América Latina: mercados de derivados y determinantes de la administración de riesgo. Panorama Económico, 11(22): 7-38. DOI: https://doi.org/10.29201/pe-ipn.v11i22.90

[12] IEA (2016). World energy outlook. OECD/IEA. DOI: https://doi.org/10.1787/be0c09b9-en

[13] Ma, H. and Wu, Y. (2009). Grey predictive on natural gas consumption and production in china. In Web Mining and Web-based Application, 2009. WMWA'09. Second Pacific-Asia Conference on, pages 9194. IEEE. DOI: https://doi.org/10.1109/wmwa.2009.26

[14] Ma, Y. and Li, Y. (2010). Analysis of the supply-demand status of China natural gas to 2020. Petroleum Science, 7(1):132-135. DOI: https://doi.org/10.1007/s12182-010-0017-9

[15] Miller, J. L. C., Olvera, V. O., et al. (2015). Reforma y nueva estructura del sector hidrocarburos en México. http://bibliodigitalibd.senado.gob.mx/handle/123456789/2191

[16] OCDE (2017). Estudios económicos de la OCDE. DOI: https://doi.org/10.1787/9789264269040-es

[17] Palacios Solano, Isaac (1987). PEMEX: ¿Los precios más bajos del mundo? Momento Económico (31). pp. 12-15. ISSN 0186-2901

[18] Pemex (1977). Anuario estadístico. PEMEX.

[19] Pemex (1990). Anuario estadístico. PEMEX.

[20] Pemex (1999). Anuario estadístico. PEMEX.

[21] SENER (2014). Prospectiva de gas natural y gas l.p. 2014-2028. México: Secretaría de Energía. 
[22] SENER (2015). Prospectiva de gas natural y gas l.p. 2015-2029. México: Secretaría de Energía.

[23] SENER (2016). Política: Pública en materia de almacenamiento de gas natural. México: Secretaría de Energía.

[24] SENER (2017). Prospectiva de gas natural 2017-2031. México: Secretaría de Energía.

[25] SENER (2018). Política Pública en materia de almacenamiento de gas natural. México: Secretaría de Energía.

[26] SIE. Sistema de Información Energética. Producción de gas natural por tipo y región. Secretaría de Energía. http://sie.energia.gob.mx/movil.do?action=back\&node=PMXC2C03

[27] Soldo, B. (2012). Forecasting natural gas consumption. Applied Energy, 92:26-37. DOI: https://doi.org/10.1016/j.apenergy.2011.11.003

[28] Szklo, A., Machado, G. and Schaeffer, R. (2007). Future oil production in Brazil-Estimates based on a Hubbert model. Energy Policy, 35 (2007), 2360-2367. DOI: https://doi.org/10.1016/j.enpol.2006.08.014

[29] Vasicek, O. (1977). An Equilibrium Characterization of the Term Structure. Journal of Financial Economics, 5(2): 177-188. DOI: https://doi.org/10.1016/0304-405X(77)90016-2

[30] Venegas-Martínez. F. (2008). Riesgos financieros y económicos: Productos Derivados y Decisiones Económicas bajo incertidumbre. Cengage Learning Editores.

[31] Xie, Y. and Li, M. (2009). Research on prediction model of natural gas consumption based on grey modeling optimized by genetic algorithm. In Control, Automation and Systems Engineering, 2009. CASE 2009. IITA International Conference on, pages 335-337. IEEE. DOI: https://doi.org/10.1109/case.2009.101 


\section{Apéndices de artículo Viabilidad de introducir contratos de derivados de gas natural en el Mercado Mexicano de Derivados.}

\section{A.1. Modelo de Grey GM(1,1)}

A.1.1. Estimación de los parámetros de la demanda total $\operatorname{GM}(1,1)$

Call: $\quad \operatorname{lm}$ (formula $=$ demanda_total $\sim$ grey_total $)$

Residuals: $\quad$ Min 1Q Median 3Q Max $-433.58-213.31-62.25142 .64493 .29$

Coefficients: Estimate Std. Error $t$ value $\operatorname{Pr}(>|t|)$ (Intercept) $4.353 \mathrm{e}-02 \quad 1.737 \mathrm{e}-03 \quad 25.05<2 \mathrm{e}-16 * * *$ grey_total $2.910 \mathrm{e}+03 \quad 1.090 \mathrm{e}+02 \quad 26.71<2 \mathrm{e}-16 * * *$

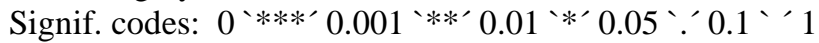

Residual standard error: 289.6 on 21 degrees of freedom

Multiple R-squared: 0.9676, Adjusted R-squared: 0.9661

F-statistic: 627.7 on 1 and $21 \mathrm{DF}, \mathrm{p}$-value: < 2.2e-16

Elaboración propia con Sofware R

A.1.2. Estimación de los parámetros de la demanda del sector eléctrico $\operatorname{GM}(1,1)$

Call: $\quad \operatorname{lm}($ formula $=$ demanda_electrica $\sim$ grey_electrica)

Residuals: Min 1Q Median 3Q Max

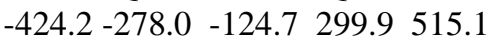

Coefficients: Estimate Std. Error $t$ value $\operatorname{Pr}(>|t|)$

(Intercept) $7.556 \mathrm{e}-02 \quad 4.706 \mathrm{e}-03 \quad 16.0552 .88 \mathrm{e}-13 * * *$

grey_electrica $7.929 \mathrm{e}+02 \quad 1.046 \mathrm{e}+02 \quad 7.5771 .95 \mathrm{e}-07 * * *$

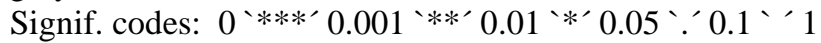

Residual standard error: 321.2 on 21 degrees of freedom

Multiple R-squared: 0.9247, Adjusted R-squared: 0.9211

F-statistic: 257.8 on 1 and 21 DF, p-value: $2.88 \mathrm{e}-13$

Elaboración propia con Sofware R

A.1.3. Estimación de los parámetros de la demanda del sector industrial GM(1,1)

Call: $\operatorname{lm}$ (formula $=$ demanda_industrial $\sim$ grey_industrial)

Residuals: Min 1Q Median 3Q Max

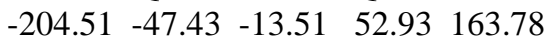

Coefficients: Estimate Std. Error t value $\operatorname{Pr}(>|t|)$ (Intercept) $\quad 0.02391 \quad 0.00275 \quad 8.695 \quad 2.11 \mathrm{e}-08 * * *$ grey_industrial $754.1216636 .6822120 .558 \quad 2.16 \mathrm{e}-15 * * *$

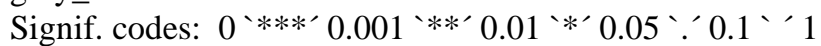

Residual standard error: 88.19 on 21 degrees of freedom

Multiple R-squared: 0.7826 , Adjusted R-squared: 0.7722

F-statistic: 75.6 on 1 and $21 \mathrm{DF}$, p-value: $2.113 \mathrm{e}-08$ Elaboración propia con Sofware $\mathrm{R}$

A.1.3. Estimación de los parámetros de la demanda del sector petrolero $\operatorname{GM}(1,1)$

Call: $\quad \operatorname{lm}($ formula $=$ demanda_petrolera $\sim$ grey_petrolera $)$

Residuals: $\quad$ Min 1Q Median 3Q Max $\begin{array}{lllll}-270.67 & -64.76 & 25.64 & 84.59 & 170.99\end{array}$

Coefficients: Estimate Std. Error $\mathrm{t}$ value $\operatorname{Pr}(>|\mathrm{t}|)$ (Intercept) $\quad 1.834 \mathrm{e}-02 \quad 1.860 \mathrm{e}-03 \quad 9.8572 .49 \mathrm{e}-09 * * *$ grey_petrolera $1.556 \mathrm{e}+03 \quad 4.854 \mathrm{e}+0132.051<2 \mathrm{e}-16 * * *$ Signif. codes: $0{ }^{` * * * *^{\prime}} 0.001^{` * *} 0.01^{\prime} *^{\prime} 0.05{ }^{\prime} \mathrm{Y}^{\prime} 0.1^{\prime}{ }^{\prime} 1$ Residual standard error: 118.8 on 21 degrees of freedom Multiple R-squared: 0.8223 , Adjusted R-squared: 0.8138 
F-statistic: 97.16 on 1 and $21 \mathrm{DF}, \mathrm{p}$-value: $2.491 \mathrm{e}-09$

Elaboración propia con Sofware $\mathrm{R}$

\section{A.2. Modelo de reversión a la media}

A.2.1. Estimación de la tasa de crecimiento de la demanda total con el modelo de reversión a la media Call: $\quad \operatorname{arima}\left(\mathrm{x}=\right.$ vasicek$\$ d \_t o t a l$, order $\left.=\mathrm{c}(1,0,0)\right)$

Coefficients: ar1 intercept

$-0.229557 \quad 0.046322$

s.e. 0.1709530 .007255

sigma $^{\wedge} 2$ estimated as 0.041353: $\log$ likelihood $=42.21386$, aic $=-3.409901$

Elaboración propia con Sofware R

A.2.2. Estimación de la tasa de crecimiento del sector eléctrico con el modelo de reversión a la media

Call: $\quad \operatorname{arima}\left(\mathrm{x}=\right.$ vasicek $\$ \mathrm{~d} \_$electrica, order $\left.=\mathrm{c}(1,0,0)\right)$

Coefficients: ar1 intercept

$0.3497 \quad 0.099416$

s.e. 0.25590 .028099

sigma 2 estimated as 0.080347: $\log$ likelihood $=26.89890$, aic $=-2.078165$

Elaboración propia con Sofware R

A.2.3. Estimación de la tasa de crecimiento del sector industrial con el modelo de reversión a la media

Call: $\quad \operatorname{arima}\left(\mathrm{x}=\right.$ vasicek $\$ d \_$industrial, order $\left.=\mathrm{c}(1,0,0)\right)$

Coefficients: ar1 intercept

$-0.26228 \quad 0.033476$

s.e. 0.2358850 .019063

sigma $^{\wedge} 2$ estimated as 0.075163: $\log$ likelihood $=28.46217$, aic $=-2.214101$

Elaboración propia con Sofware R

A.2.4. Estimación de la tasa de crecimiento del sector petrolero con el modelo de reversión a la media

Call: $\quad \operatorname{arima}\left(\mathrm{x}=\right.$ vasicek $\$ \mathrm{~d} \_$petrolera, order $\left.=\mathrm{c}(1,0,0)\right)$

Coefficients: ar1 intercept

$-0.22465 \quad 0.017673$

s.e. 0.1841150 .008649

sigma $^{\wedge} 2$ estimated as 0.051099: $\log$ likelihood $=37.34776$, aic $=-2.986762$

Elaboración propia con Sofware R

Tabla 3. Pronóstico de la tasa de crecimiento de la demanda total de gas natural

\begin{tabular}{|c|c|c|c|c|c|c|c|c|}
\hline & $\mathrm{TC}$ & $\mathrm{r}_{\mathrm{t}}+1-\mathrm{r}_{\mathrm{t}}$ & $\mathrm{a}\left(\mathrm{b}-\mathrm{r}_{\mathrm{t}}\right)$ & $\mathrm{r}_{\mathrm{t}}+1-\mathrm{r}_{\mathrm{t}}-\mathrm{a}\left(\mathrm{b}-\mathrm{r}_{\mathrm{t}}\right)$ & $\mathrm{Z}$ & $\mu$ & $\sigma^{2}$ & Pronóstico \\
\hline 1994 & 0.0444 & & & & & & & \\
\hline 1995 & 0.0003 & -0.0441 & 0.1005 & -0.1446 & -3.8511 & 0.0267 & -0.0042 & 0.0225 \\
\hline 1996 & 0.0645 & 0.0642 & -0.0327 & 0.0969 & -0.4786 & 0.0455 & -0.0005 & 0.0450 \\
\hline 1997 & 0.0741 & 0.0096 & 0.0345 & -0.0249 & 2.0855 & 0.0483 & 0.0023 & 0.0506 \\
\hline 1998 & 0.0915 & 0.0173 & 0.0250 & -0.0077 & 2.2494 & 0.0534 & 0.0025 & 0.0559 \\
\hline 1999 & -0.0121 & -0.1036 & 0.1737 & -0.2772 & -20.2068 & 0.0231 & -0.0222 & 0.0009 \\
\hline 2000 & 0.0980 & 0.1101 & -0.0890 & 0.1991 & -9.3198 & 0.0553 & -0.0103 & 0.0450 \\
\hline 2001 & 0.0063 & -0.0917 & 0.1590 & -0.2507 & -16.1136 & 0.0285 & -0.0177 & 0.0108 \\
\hline 2002 & 0.1333 & 0.1270 & -0.1098 & 0.2368 & -14.1285 & 0.0656 & -0.0156 & 0.0501 \\
\hline 2003 & 0.0956 & -0.0376 & 0.0926 & -0.1302 & -2.6921 & 0.0546 & -0.0030 & 0.0517 \\
\hline 2004 & 0.0636 & -0.0321 & 0.0858 & -0.1178 & -1.7924 & 0.0452 & -0.0020 & 0.0433 \\
\hline
\end{tabular}




\begin{tabular}{|l|c|c|c|c|c|c|c|c|}
\hline 2005 & -0.0155 & -0.0790 & 0.1435 & -0.2225 & -12.2124 & 0.0221 & -0.0134 & 0.0087 \\
\hline 2006 & 0.1151 & 0.1305 & -0.1142 & 0.2447 & -15.2415 & 0.0603 & -0.0168 & 0.0435 \\
\hline 2007 & 0.0446 & -0.0705 & 0.1330 & -0.2034 & -9.8353 & 0.0397 & -0.0108 & 0.0289 \\
\hline 2008 & 0.0310 & -0.0135 & 0.0630 & -0.0765 & 0.5549 & 0.0357 & 0.0006 & 0.0363 \\
\hline 2009 & -0.0009 & -0.0320 & 0.0857 & -0.1177 & -1.7813 & 0.0264 & -0.0020 & 0.0244 \\
\hline 2010 & 0.0388 & 0.0398 & -0.0026 & 0.0423 & 1.7434 & 0.0380 & 0.0019 & 0.0399 \\
\hline 2011 & 0.0270 & -0.0118 & 0.0608 & -0.0726 & 0.7265 & 0.0346 & 0.0008 & 0.0354 \\
\hline 2012 & 0.0255 & -0.0015 & 0.0482 & -0.0497 & 1.5452 & 0.0341 & 0.0017 & 0.0358 \\
\hline 2013 & 0.0410 & 0.0155 & 0.0273 & -0.0118 & 2.2261 & 0.0387 & 0.0025 & 0.0411 \\
\hline 2014 & 0.0370 & -0.0041 & 0.0513 & -0.0554 & 1.3708 & 0.0375 & 0.0015 & 0.0390 \\
\hline 2015 & 0.0409 & 0.0039 & 0.0415 & -0.0376 & 1.8538 & 0.0386 & 0.0020 & 0.0407 \\
\hline 2016 & 0.0153 & -0.0256 & 0.0778 & -0.1034 & -0.8617 & 0.0311 & -0.0009 & 0.0302 \\
\hline 2017 & 0.0382 & 0.0229 & 0.0181 & 0.0048 & 2.2598 & 0.0378 & 0.0025 & 0.0403 \\
\hline 2018 & 0.0403 & 0.0021 & 0.0437 & -0.0416 & 1.7600 & 0.0384 & 0.0019 & 0.0404 \\
\hline 2019 & 0.0404 & 0.0001 & 0.0462 & -0.0462 & 1.6432 & 0.0385 & 0.0018 & 0.0403 \\
\hline 2020 & 0.0403 & -0.0001 & 0.0465 & -0.0466 & 1.6327 & 0.0384 & 0.0018 & 0.0402 \\
\hline 2021 & 0.0402 & 0.0000 & 0.0464 & -0.0464 & 1.6367 & 0.0384 & 0.0018 & 0.0402 \\
\hline 2022 & 0.0402 & 0.0000 & 0.0463 & -0.0463 & 1.6388 & 0.0384 & 0.0018 & 0.0402 \\
\hline 2023 & 0.0402 & 0.0000 & 0.0463 & -0.0463 & 1.6393 & 0.0384 & 0.0018 & 0.0402 \\
\hline 2024 & 0.0402 & 0.0000 & 0.0463 & -0.0463 & 1.6393 & 0.0384 & 0.0018 & 0.0402 \\
\hline 2025 & 0.0402 & 0.0000 & 0.0463 & -0.0463 & 1.6393 & 0.0384 & 0.0018 & 0.0402 \\
\hline
\end{tabular}

Elaboración propia con Sofware Excel

Tabla 4. Pronóstico de la tasa de crecimiento de la demanda eléctrica

\begin{tabular}{|c|c|c|c|c|c|c|c|c|}
\hline & $\mathrm{TC}$ & $\mathrm{r}_{\mathrm{t}}+1-\mathrm{r}_{\mathrm{t}}$ & $\mathrm{a}\left(\mathrm{b}-\mathrm{r}_{\mathrm{t}}\right)$ & $\mathrm{r}_{\mathrm{t}}+1-\mathrm{r}_{\mathrm{t}}-\mathrm{a}\left(\mathrm{b}-\mathrm{r}_{\mathrm{t}}\right)$ & $\mathrm{Z}$ & $\mu$ & $\sigma^{2}$ & Pronóstico \\
\hline 1994 & 0.1758 & & & & & & & \\
\hline 1995 & 0.0777 & -0.0981 & 0.1632 & -0.2613 & -3.6853 & 0.1136 & -0.0203 & 0.0934 \\
\hline 1996 & 0.0121 & -0.0657 & 0.1421 & -0.2078 & -1.7424 & 0.0794 & -0.0096 & 0.0698 \\
\hline 1997 & 0.0956 & 0.0835 & 0.0451 & 0.0384 & 1.4882 & 0.1229 & 0.0082 & 0.1311 \\
\hline 1998 & 0.1566 & 0.0611 & 0.0597 & 0.0014 & 1.6023 & 0.1548 & 0.0088 & 0.1636 \\
\hline 1999 & 0.0873 & -0.0693 & 0.1445 & -0.2138 & -1.9388 & 0.1186 & -0.0107 & 0.1080 \\
\hline 2000 & 0.2312 & 0.1438 & 0.0058 & 0.1380 & 0.1265 & 0.1936 & 0.0007 & 0.1943 \\
\hline 2001 & 0.1436 & -0.0875 & 0.1564 & -0.2439 & -3.0057 & 0.1480 & -0.0165 & 0.1315 \\
\hline 2002 & 0.2981 & 0.1544 & -0.0011 & 0.1556 & -0.2717 & 0.2285 & -0.0015 & 0.2270 \\
\hline 2003 & 0.2220 & -0.0760 & 0.1489 & -0.2249 & -2.3169 & 0.1889 & -0.0127 & 0.1761 \\
\hline 2004 & 0.1175 & -0.1045 & 0.1674 & -0.2720 & -4.1277 & 0.1343 & -0.0227 & 0.1117 \\
\hline 2005 & -0.0180 & -0.1355 & 0.1876 & -0.3231 & -6.4850 & 0.0637 & -0.0356 & 0.0280 \\
\hline 2006 & 0.1869 & 0.2049 & -0.0340 & 0.2389 & -2.8182 & 0.1705 & -0.0155 & 0.1551 \\
\hline 2007 & 0.1073 & -0.0796 & 0.1512 & -0.2308 & -2.5246 & 0.1290 & -0.0139 & 0.1151 \\
\hline 2008 & 0.0560 & -0.0513 & 0.1328 & -0.1841 & -1.0228 & 0.1023 & -0.0056 & 0.0966 \\
\hline 2009 & 0.0497 & -0.0063 & 0.1035 & -0.1098 & 0.6693 & 0.0990 & 0.0037 & 0.1027 \\
\hline 2010 & 0.0012 & -0.0485 & 0.1310 & -0.1795 & -0.8922 & 0.0737 & -0.0049 & 0.0688 \\
\hline 2011 & 0.0518 & 0.0506 & 0.0665 & -0.0159 & 1.5829 & 0.1001 & 0.0087 & 0.1088 \\
\hline 2012 & 0.0075 & -0.0443 & 0.1282 & -0.1725 & -0.7036 & 0.0770 & -0.0039 & 0.0731 \\
\hline 2013 & 0.0679 & 0.0604 & 0.0601 & 0.0003 & 1.6025 & 0.1085 & 0.0088 & 0.1173 \\
\hline 2014 & 0.0535 & -0.0144 & 0.1088 & -0.1232 & 0.4261 & 0.1009 & 0.0023 & 0.1033 \\
\hline
\end{tabular}


REMEF (The Mexican Journal of Economics and Finance)

Viabilidad de introducir contratos de derivados de gas natural en el Mercado

Mexicano de Derivados: Un enfoque Hubbert-Grey

\begin{tabular}{|l|l|l|l|l|l|l|l|l|}
\hline 2015 & 0.0849 & 0.0315 & 0.0789 & -0.0475 & 1.4280 & 0.1174 & 0.0079 & 0.1252 \\
\hline 2016 & 0.0213 & -0.0636 & 0.1408 & -0.2044 & -1.6344 & 0.0842 & -0.0090 & 0.0752 \\
\hline 2017 & 0.0752 & 0.0539 & 0.0643 & -0.0104 & 1.5941 & 0.1123 & 0.0088 & 0.1211 \\
\hline 2018 & 0.1211 & 0.0459 & 0.0696 & -0.0237 & 1.5590 & 0.1362 & 0.0086 & 0.1448 \\
\hline 2019 & 0.1448 & 0.0237 & 0.0840 & -0.0602 & 1.3214 & 0.1486 & 0.0073 & 0.1558 \\
\hline 2020 & 0.0465 & -0.0983 & 0.1634 & -0.2616 & -3.7001 & 0.0973 & -0.0203 & 0.0770 \\
\hline 2021 & 0.0770 & 0.0305 & 0.0796 & -0.0491 & 1.4158 & 0.1132 & 0.0078 & 0.1210 \\
\hline 2022 & 0.1210 & 0.0440 & 0.0708 & -0.0267 & 1.5471 & 0.1362 & 0.0085 & 0.1447 \\
\hline 2023 & 0.1447 & 0.0237 & 0.0840 & -0.0603 & 1.3208 & 0.1485 & 0.0073 & 0.1558 \\
\hline 2024 & 0.1558 & 0.0111 & 0.0922 & -0.0811 & 1.0934 & 0.1543 & 0.0060 & 0.1603 \\
\hline 2025 & 0.1603 & 0.0045 & 0.0964 & -0.0919 & 0.9483 & 0.1567 & 0.0052 & 0.1619 \\
\hline
\end{tabular}

Elaboración propia con Sofware Excel

Tabla 5. Pronóstico de la tasa de crecimiento de la demanda industrial

\begin{tabular}{|c|c|c|c|c|c|c|c|c|}
\hline & $\mathrm{TC}$ & $\mathrm{r}_{\mathrm{t}}+1-\mathrm{r}_{\mathrm{t}}$ & $a\left(b-r_{t}\right)$ & $r_{t}+1-r_{t}-a\left(b-r_{t}\right)$ & $\mathrm{Z}$ & $\mu$ & $\sigma^{2}$ & Pronóstico \\
\hline 1994 & 0.0134 & & & & & & & \\
\hline 1995 & 0.0714 & 0.0579 & -0.0396 & 0.0976 & 0.8264 & 0.0392 & 0.0030 & 0.0422 \\
\hline 1996 & 0.0834 & 0.0120 & 0.0183 & -0.0063 & 1.6656 & 0.0426 & 0.0061 & 0.0487 \\
\hline 1997 & 0.0234 & -0.0600 & 0.1092 & -0.1691 & -0.8620 & 0.0256 & -0.0032 & 0.0225 \\
\hline 1998 & 0.0870 & 0.0636 & -0.0467 & 0.1103 & 0.5924 & 0.0436 & 0.0022 & 0.0458 \\
\hline 1999 & 0.0626 & -0.0243 & 0.0642 & -0.0885 & 0.9753 & 0.0367 & 0.0036 & 0.0403 \\
\hline 2000 & -0.0037 & -0.0664 & 0.1173 & -0.1837 & -1.3159 & 0.0180 & -0.0048 & 0.0131 \\
\hline 2001 & -0.1773 & -0.1735 & 0.2525 & -0.4261 & -14.3970 & -0.0312 & -0.0527 & -0.0839 \\
\hline 2002 & 0.1515 & 0.3288 & -0.3815 & 0.7103 & -42.9803 & 0.0619 & -0.1574 & -0.0955 \\
\hline 2003 & -0.0429 & -0.1944 & 0.2789 & -0.4733 & -18.1553 & 0.0069 & -0.0665 & -0.0596 \\
\hline 2004 & 0.0352 & 0.0781 & -0.0651 & 0.1432 & -0.1444 & 0.0290 & -0.0005 & 0.0284 \\
\hline 2005 & -0.0223 & -0.0574 & 0.1060 & -0.1634 & -0.6933 & 0.0127 & -0.0025 & 0.0102 \\
\hline 2006 & 0.0842 & 0.1065 & -0.1010 & 0.2075 & -2.1412 & 0.0429 & -0.0078 & 0.0350 \\
\hline 2007 & 0.0257 & -0.0586 & 0.1074 & -0.1660 & -0.7686 & 0.0263 & -0.0028 & 0.0235 \\
\hline 2008 & -0.0130 & -0.0386 & 0.0822 & -0.1209 & 0.3758 & 0.0153 & 0.0014 & 0.0167 \\
\hline 2009 & -0.1108 & -0.0979 & 0.1570 & -0.2549 & -4.0795 & -0.0124 & -0.0149 & -0.0273 \\
\hline 2010 & 0.1550 & 0.2658 & -0.3020 & 0.5679 & -26.8694 & 0.0629 & -0.0984 & -0.0355 \\
\hline 2011 & 0.0711 & -0.0839 & 0.1394 & -0.2233 & -2.7444 & 0.0391 & -0.0101 & 0.0291 \\
\hline 2012 & 0.0459 & -0.0252 & 0.0653 & -0.0905 & 0.9446 & 0.0320 & 0.0035 & 0.0355 \\
\hline 2013 & 0.0498 & 0.0040 & 0.0285 & -0.0245 & 1.6158 & 0.0331 & 0.0059 & 0.0390 \\
\hline 2014 & 0.0593 & 0.0095 & 0.0215 & -0.0120 & 1.6564 & 0.0358 & 0.0061 & 0.0419 \\
\hline 2015 & 0.0478 & -0.0115 & 0.0480 & -0.0596 & 1.3552 & 0.0325 & 0.0050 & 0.0375 \\
\hline 2016 & 0.0784 & 0.0306 & -0.0051 & 0.0357 & 1.5560 & 0.0412 & 0.0057 & 0.0469 \\
\hline 2017 & 0.0469 & -0.0315 & 0.0732 & -0.1047 & 0.6985 & 0.0323 & 0.0026 & 0.0348 \\
\hline 2018 & 0.0348 & -0.0121 & 0.0487 & -0.0607 & 1.3426 & 0.0289 & 0.0058 & 0.0346 \\
\hline 2019 & 0.0289 & -0.0060 & 0.0410 & -0.0470 & 1.4738 & 0.0272 & 0.0054 & 0.0326 \\
\hline 2020 & 0.0326 & 0.0037 & 0.0288 & -0.0251 & 1.6134 & 0.0282 & 0.0059 & 0.0341 \\
\hline 2021 & 0.0341 & 0.0016 & 0.0315 & -0.0299 & 1.5898 & 0.0287 & 0.0058 & 0.0345 \\
\hline 2022 & 0.0345 & 0.0004 & 0.0330 & -0.0327 & 1.5746 & 0.0288 & 0.0058 & 0.0345 \\
\hline 2023 & 0.0345 & 0.0000 & 0.0334 & -0.0334 & 1.5705 & 0.0288 & 0.0058 & 0.0345 \\
\hline
\end{tabular}




\begin{tabular}{|l|l|l|l|l|l|l|l|l|}
\hline 2024 & 0.0345 & 0.0000 & 0.0335 & -0.0335 & 1.5699 & 0.0288 & 0.0057 & 0.0345 \\
\hline 2025 & 0.0345 & 0.0000 & 0.0335 & -0.0335 & 1.5699 & 0.0288 & 0.0057 & 0.0345 \\
\hline
\end{tabular}

Elaboración propia con Sofware Excel

Tabla 6. Pronóstico de la tasa de crecimiento de la demanda petrolera.

\begin{tabular}{|c|c|c|c|c|c|c|c|c|}
\hline $\mathrm{TC}$ & $r_{t}+1-r$ & $a\left(b-r_{t}\right)$ & $r_{t}+1-r_{t}-a\left(b-r_{t}\right)$ & $\mathrm{Z}$ & $\mu$ & $\sigma^{2}$ & Pronóstico & $\mathrm{TC}$ \\
\hline 1994 & 0.0230 & & & & & & & \\
\hline 1995 & -0.0630 & -0.0860 & 0.1229 & -0.2089 & -6.3054 & -0.0083 & -0.0106 & -0.0189 \\
\hline 1996 & 0.0770 & 0.1400 & -0.1537 & 0.2937 & -14.4686 & 0.0328 & -0.0243 & 0.0085 \\
\hline 1997 & 0.0968 & 0.0198 & -0.0066 & 0.0264 & 1.9218 & 0.0386 & 0.0032 & 0.0419 \\
\hline 1998 & 0.0761 & -0.0207 & 0.0430 & -0.0637 & 1.2786 & 0.0326 & 0.0021 & 0.0347 \\
\hline 1999 & -0.0950 & -0.1711 & 0.2272 & -0.3983 & -28.3294 & -0.0177 & -0.0476 & -0.0653 \\
\hline 2000 & 0.0974 & 0.1923 & -0.2179 & 0.4102 & -30.1780 & 0.0388 & -0.0507 & -0.0119 \\
\hline 2001 & 0.0302 & -0.0672 & 0.0999 & -0.1671 & -3.2950 & 0.0191 & -0.0055 & 0.0135 \\
\hline 2002 & 0.0221 & -0.0080 & 0.0275 & -0.0356 & 1.8128 & 0.0167 & 0.0030 & 0.0197 \\
\hline 2003 & 0.0671 & 0.0449 & -0.0373 & 0.0823 & 0.7586 & 0.0299 & 0.0013 & 0.0312 \\
\hline 2004 & 0.0273 & -0.0398 & 0.0664 & -0.1062 & -0.1040 & 0.0182 & -0.0002 & 0.0180 \\
\hline 2005 & -0.0110 & -0.0383 & 0.0646 & -0.1028 & 0.0297 & 0.0070 & 0.0000 & 0.0070 \\
\hline 2006 & 0.0639 & 0.0748 & -0.0740 & 0.1488 & -2.1860 & 0.0290 & -0.0037 & 0.0253 \\
\hline 2007 & -0.0159 & -0.0797 & 0.1153 & -0.1950 & -5.2275 & 0.0055 & -0.0088 & -0.0032 \\
\hline 2008 & 0.0233 & 0.0392 & -0.0303 & 0.0695 & 1.1305 & 0.0170 & 0.0019 & 0.0189 \\
\hline 2009 & -0.0117 & -0.0351 & 0.0606 & -0.0956 & 0.3028 & 0.0067 & 0.0005 & 0.0073 \\
\hline 2010 & 0.0405 & 0.0523 & -0.0463 & 0.0986 & 0.1932 & 0.0221 & 0.0003 & 0.0224 \\
\hline 2011 & -0.0225 & -0.0631 & 0.0949 & -0.1579 & -2.7231 & 0.0036 & -0.0046 & -0.0010 \\
\hline 2012 & 0.0397 & 0.0622 & -0.0585 & 0.1208 & -0.7386 & 0.0219 & -0.0012 & 0.0206 \\
\hline 2013 & -0.0004 & -0.0401 & 0.0668 & -0.1069 & -0.1321 & 0.0101 & -0.0002 & 0.0099 \\
\hline 2014 & 0.0015 & 0.0018 & 0.0154 & -0.0136 & 2.0199 & 0.0106 & 0.0034 & 0.0140 \\
\hline 2015 & -0.0332 & -0.0347 & 0.0601 & -0.0948 & 0.3329 & 0.0004 & 0.0006 & 0.0010 \\
\hline 2016 & -0.0355 & -0.0023 & 0.0204 & -0.0227 & 1.9564 & -0.0002 & 0.0033 & 0.0031 \\
\hline 2017 & 0.0031 & 0.0385 & -0.0295 & 0.0680 & 1.1688 & 0.0111 & 0.0020 & 0.0131 \\
\hline 2018 & 0.0131 & 0.0100 & 0.0054 & 0.0046 & 2.0512 & 0.0140 & 0.0034 & 0.0174 \\
\hline 2019 & 0.0140 & 0.0010 & 0.0165 & -0.0155 & 2.0092 & 0.0143 & 0.0034 & 0.0177 \\
\hline 2020 & 0.0177 & 0.0037 & 0.0132 & -0.0095 & 2.0378 & 0.0154 & 0.0034 & 0.0188 \\
\hline 2021 & 0.0188 & 0.0011 & 0.0163 & -0.0152 & 2.0111 & 0.0157 & 0.0034 & 0.0191 \\
\hline 2022 & 0.0191 & 0.0003 & 0.0173 & -0.0170 & 1.9996 & 0.0158 & 0.0034 & 0.0192 \\
\hline 2023 & 0.0192 & 0.0001 & 0.0176 & -0.0175 & 1.9963 & 0.0158 & 0.0034 & 0.0192 \\
\hline 2024 & 0.0192 & 0.0000 & 0.0177 & -0.0176 & 1.9956 & 0.0158 & 0.0034 & 0.0192 \\
\hline 2025 & 0.0192 & 0.0000 & 0.0177 & -0.0177 & 1.9954 & 0.0158 & 0.0034 & 0.0192 \\
\hline
\end{tabular}

Elaboración propia con Sofware Excel

\section{A.3 Modelo de Hubbert}

Call: $\quad \operatorname{lm}($ formula $=$ hub $\sim$ hubbert $)$

Residuals: $\quad$ Min 1Q Median 3Q Max

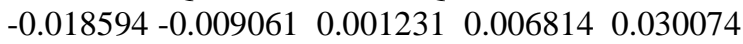

Coefficients: Estimate Std. Error t value $\operatorname{Pr}(>|t|)$ 
(Intercept) $0.1236100 \quad 0.0041657 \quad 33.35<2 \mathrm{e}-16 * * *$

hubbert $-0.00125040 .0000669-22.13<2 \mathrm{e}-16 * * *$

$---$

Signif. codes: $0{ }^{`} * * *^{\prime} 0.001^{`} * *^{\prime} 0.01^{`} *^{\prime} 0.05{ }^{\prime} 0.1^{`}{ }^{\prime} 1$

Residual standard error: 0.01107 on 67 degrees of freedom

Multiple R-squared: 0.8796, Adjusted R-squared: 0.8779

F-statistic: 489.7 on 1 and $67 \mathrm{DF}$, p-value: $<2.2 \mathrm{e}-16$

Elaboración propia con Sofware R

\section{A.4. Simulación Montecarlo de la VME}

$>\mathrm{mu}<-73.31342143$

$>$ sigma $<-0.057467269$

$>$ alpha $<-0.01$

$>$ conf <- 1 - alpha \# VaR al $99 \%$

$>$ qnorm(alpha)

[1] -2.326348

$>$ qnorm(alpha, mean $=\mathrm{mu}, \mathrm{sd}=$ sigma $)$

[1] 73.17973

$>\operatorname{pnorm}(73.17973$, mean $=\mathrm{mu}, \mathrm{sd}=\operatorname{sigma})$

[1] 0.009998808

$>$ pnorm(qnorm $(0.01))$

[1] 0.01

$>\operatorname{sim}<-\operatorname{rnorm}(10000$, mean $=\mathrm{mu}, \mathrm{sd}=$ sigma $)$

$>$ quantile $(\operatorname{sim}, \mathrm{c}(.01, .5))$ $1 \% \quad 50 \%$

73.1765273 .31420

Elaboración propia con Sofware R

\section{A.5. Simulación Montecarlo del precio Spot para el contrato de futuros de gas natural}

$>$ norm.simulated <- replicate $(\mathrm{n}=1000, \operatorname{rnorm}(\mathrm{n}=8$, mean $=73.31342143, \mathrm{sd}=0.057467269))$

$>$ sd(apply(norm.simulated,2,mean))

[1] 0.02045131

$>$ mean(apply(norm.simulated,2,mean))

[1] 73.31294

$>$ hist(apply(norm.simulated,2,mean))

$>$ norm.simulated

Elaboración propia con Sofware R

\section{A.6. Opción call por el método Cox, Ross y Rubinstein}

Title: CRR Binomial Tree Option

Call: CRRBinomialTreeOption(TypeFlag = "ce", $\mathrm{S}=74.154, \mathrm{X}=74.2787$, Time $=365 / 52, \mathrm{r}=0.0812$, $\mathrm{b}=0$, sigma

$=0.05747, \mathrm{n}=7$ )

Parameters: Value:

TypeFlag ce

S 74.154

X $\quad 74.2787$

Time $\quad 7.01923076923077$

r $\quad 0.0812$

b 0

sigma $\quad 0.05747$

n 7 
Option Price: 2.603768

Elaboración propia con Sofware R

\section{A.7. Opción put por el método Cox, Ross y Rubinstein}

Title: CRR Binomial Tree Option

Call: CRRBinomialTreeOption(TypeFlag $=$ "pe", $\mathrm{S}=74.154, \mathrm{X}=74.2787$, Time $=365 / 52, \mathrm{r}=0.0812, \mathrm{~b}=0$, sigma $=0.05747, \mathrm{n}=7$ )

Parameters: Value:

TypeFlag pe

S 74.154

X $\quad 74.2787$

Time $\quad 7.01923076923077$

r $\quad 0.0812$

b $\quad 0$

sigma $\quad 0.05747$

n 7

Option Price:

2.674291

Elaboración propia con Sofware $\mathrm{R}$ 